\title{
MASS TRANSPORT AND TURBULENCE IN GRAVITATIONALLY UNSTABLE DISK GALAXIES. I. THE CASE OF PURE SELF-GRAVITY
}

\author{
Nathan J. Goldbaum ${ }^{1,2}$, Mark R. Krumholz ${ }^{2}$, and John C. Forbes ${ }^{2}$ \\ ${ }^{1}$ National Center for Supercomputing Applications, University of Illinois, 1205 West Clark Street, Urbana-Champaign, Illinois 61820, USA \\ ${ }^{2}$ Department of Astronomy \& Astrophysics, University of California, Santa Cruz, CA 95064, USA \\ Received 2015 July 10; accepted 2015 October 28; published 2015 November 25
}

\begin{abstract}
The role of gravitational instability-driven turbulence in determining the structure and evolution of disk galaxies, and the extent to which gravity rather than feedback can explain galaxy properties, remains an open question. To address it, we present high-resolution adaptive mesh refinement simulations of Milky Way-like isolated disk galaxies, including realistic heating and cooling rates and a physically motivated prescription for star formation, but no form of star formation feedback. After an initial transient, our galaxies reach a state of fully nonlinear gravitational instability. In this state, gravity drives turbulence and radial inflow. Despite the lack of feedback, the gas in our galaxy models shows substantial turbulent velocity dispersions, indicating that gravitational instability alone may be able to power the velocity dispersions observed in nearby disk galaxies on $100 \mathrm{pc}$ scales. Moreover, the rate of mass transport produced by this turbulence approaches $\sim 1 M_{\odot} \mathrm{yr}^{-1}$ for Milky Way-like conditions, sufficient to fully fuel star formation in the inner disks of galaxies. In a companion paper, we add feedback to our models, and use the comparison between the two cases to understand which galaxy properties depend sensitively on feedback and which can be understood as the product of gravity alone. All of the code, initial conditions, and simulation data for our model are publicly available.
\end{abstract}

Key words: galaxies: evolution - galaxies: kinematics and dynamics - galaxies: spiral ISM: kinematics and dynamics - ISM: structure

\section{INTRODUCTION}

\subsection{Gravitational Instability as a Driver of Galactic Evolution}

Until the past few years, most theoretical work on galaxy evolution has focused on reproducing observed correlations in the bulk properties of galaxies, such as the stellar mass-halo mass relation (e.g., Behroozi et al. 2013; Moster et al. 2013), the stellar mass-star formation relation (e.g., Daddi et al. 2007; Elbaz et al. 2007; Noeske et al. 2007), and the mass-metallicity relation (e.g., Pilyugin et al. 2004; Tremonti et al. 2004). In such correlations, galaxies are treated as single points, and theoretical models have been largely content to treat them as such. In these models, the behavior of galaxies is almost entirely dictated by a balance between cosmological accretion and feedback (e.g., Bouché et al. 2010; Lilly et al. 2013; Forbes et al. 2014b; Mitra et al. 2015), and for this reason most theoretical attention has been focused on stellar feedback as the dominant driver of galaxy evolution.

The recent availability of large samples of spatially resolved maps of gas, metals, and star formation in nearby galaxies has opened up a new frontier in the study of galaxies: explaining the radial distribution of these quantities. Observational studies conducted to date have turned up puzzling facts that demand explanation, which cannot obviously be explained simply by a local balance of accretion versus feedback at all locations in a galaxy. Instead, these observations point to a potentially significant role for radial redistribution of material as a key process for understanding the evolution and growth of galaxies over cosmological timescales.

The first observational puzzle concerns the radial distribution of gas and star formation in present-day disk galaxies. Observations show that the neutral gas in such galaxies is distributed with an approximately universal exponential profile, with typical gas scale lengths of $\sim 0.5 r_{25}$, where $r_{25}$ is the optical radius of the galaxy (Regan et al. 2001; Schruba et al. 2011; Bigiel \& Blitz 2012). The gas is dominated by $\mathrm{H}_{2}$ inside $\sim 0.4 r_{25}$, while $\mathrm{H}$ I predominates at larger radii (e.g., Leroy et al. 2008). The radial distribution of star formation is, to first approximation, simply linearly proportional to the $\mathrm{H}_{2}$ distribution (Bigiel et al. 2008; Schruba et al. 2011; Leroy et al. 2013), so star formation is much more radially concentrated than the total neutral gas, and predominantly occurs inside $\sim 0.5 r_{25}$. In such regions, the time required to convert all the available gas to stars is $\sim 2 \mathrm{Gyr}$, much less than a Hubble time (Bigiel et al. 2008; Leroy et al. 2013). Thus the inner parts of galaxies will become gas-depleted and cease star formation unless fresh gas is supplied at an equal rate, which for $L_{*}$ galaxies is a few $M_{\odot} \mathrm{yr}^{-1}$ (Kennicutt \& Evans 2012). While some disk galaxies, including the Milky Way, do have inner gas holes and quenched central star formation, such systems appear to be the exception rather than the rule among the local disk galaxy population (Bigiel \& Blitz 2012).

How the fresh gas responsible for the lack of quenched centers can be explained is an unsolved problem. While condensation of gas from the hot halo (Marinacci et al. 2010; Fraternali et al. 2013; Hobbs et al. 2013) will supply gas in a centrally concentrated manner, direct accretion of cold gas from the intergalactic medium (e.g., Kereš et al. 2005; Dekel et al. 2009a) primarily deposits gas at large galactocentric radii, far from the actively star-forming regions that need to be refueled. This can lead to build-up of gas at large radii (c.f. Dutton 2012). Gas recycling from evolved stellar populations can potentially provide some of the required mass (Leitner \& Kravtsov 2011) at small galactocentric radii, but, in addition to uncertainties about whether this channel provides enough mass and is consistent with various chemical evolution constraints, the radial distribution of recycled gas has not been explored. In summary, the lack of quenched, gas-depleted centers in local 
disk galaxies seems to require some form of gas redistribution within the disk, for which gravitational instability is an obvious candidate.

A second surprising observation regarding the radial distribution of gas concerns gas velocity dispersions. $\mathrm{H}$ i lines in disk galaxies show super-thermal velocity dispersions of $\sim 10 \mathrm{~km} \mathrm{~s}^{-1}$ (e.g., van Zee \& Bryant 1999; Petric \& Rupen 2007; Tamburro et al. 2009; Ianjamasimanana et al. 2012, 2015). Velocity dispersions are highest toward the centers of galaxies, but they decline only shallowly with radius, and remain superthermal even well outside $r_{25}$. While star formation feedback alone appears able to drive the observed velocity dispersion in inner disks, the same is not true outside $r_{25}$, where the star formation rate drops precipitously but the velocity dispersion does not (Tamburro et al. 2009; Ianjamasimanana et al. 2015). Dwarf galaxies represent an extreme in this regard: due to their very low rates of star formation, supernova feedback cannot plausibly provide enough energy to drive the observed turbulence anywhere within them (Stilp et al. 2013). Nor do magneto-rotational or thermal instability appear to be sufficient (Kim et al. 2003; Piontek \& Ostriker 2004, 2005, 2007; Yang et al. 2007). Again, gravitational instability is an obvious candidate to drive the turbulence in the weakly star-forming portions of galaxies (although see Elmegreen \& Hunter 2015, who show that gravitational instability is not globally important in very lowmass dwarfs).

A final surprising observation concerns the metallicity distributions of galaxies. Observations of disk galaxies show that they have slightly negative gas-phase metallicity gradients inside $r_{25}$ (e.g., Vila-Costas \& Edmunds 1992; Pilyugin et al. 2004; Henry et al. 2010; Balser et al. 2011; Ho et al. 2015), and nearly flat distributions of metallicity outside $r_{25}$ (Bresolin et al. 2009, 2012; Werk et al. 2011). Dwarf galaxies show no metallicity gradients at all (Croxall et al. 2009). While the gradients in inner disks might be explicable simply via a balance between inflow, outflow, and star formation (e.g., see Portinari \& Chiosi 2000 and Spitoni \& Matteucci 2011 versus Ho et al. 2015), this is not the case for the flat gradients in outer disks. In these strongly gasdominated regions, the metallicity should simply scale with the stellar mass fraction, regardless of the presence of either inflow or outflow. While the stellar mass fraction changes sharply with galactocentric radius outside $r_{25}$, the metallicity does not, a finding that seems extremely difficult to explain without invoking some form of metal redistribution. As with the previous two results, gravitational instability seems a candidate redistribution mechanism worthy of further exploration.

\subsection{Theoretical Studies of Gravitational Instability}

Given the likely importance of gravitational instability as a driver of galaxy evolution, it is not surprising that there have been a number of theoretical studies devoted to it. Some of the earliest were one-dimensional (1D) models by Lin \& Pringle (1987a, 1987b), Olivier et al. (1991), and Ferguson \& Clarke (2001), who argued that the observed exponential distribution of stars in galactic disks could only be understood if gas undergoes significant viscous transport on a timescale comparable to the timescale over which the stellar disk forms. These models assumed a fixed dimensionless viscosity, but Krumholz \& Burkert (2010; generalizing earlier work by Bertin \& Lodato
1999 in the context of Keplerian disks with no stars) showed that the viscosity and thus the rates of mass and angular momentum transport could be computed self-consistently by balancing energy loss against turbulence generation by gravitational instability.

Building on this work, (Forbes et al. 2012, 2014a) argue that the observed structure of star forming disks is a natural result of an equilibrium between gravitational instability, accretion, and star formation. Cosmological accretion brings gas to the disk outskirts, where the gas is in general stable to collapse. Over the course of a few rotation periods, gravitational instability drives torques that tend to move gas to smaller galactocentric radii. The inward flow of gas feeds active ongoing star formation in the inner disk, such that the total star formation in the inner disk is ultimately modulated by the accretion of gas at large galactocentric radii. In these models the bulk of the disk is at all times near $Q_{\text {total }} \sim 1$.

Our motivation for three-dimensional (3D) simulations is that, while the 1D models are instructive and useful for exploring parameter space quickly, they have substantial limitations. The gas and stars both contribute to the self-gravity of the disk, so the degree of gravitational instability of both components must be considered (Lin \& Shu 1966; Jog \& Solomon 1984a, 1984b; Bertin \& Romeo 1988; Wang \& Silk 1994; Romeo \& Wiegert 2011), in the form of the Toomre (1964) stability criterion. While this is possible in 1D models, it requires strong assumptions about the vertical and azimuthal structure of the disk, and about the response of each component. Moreover, material at a given radius is only allowed to move inward or outward at one bulk velocity. Properly modeling the full nonlinear multi-component gravitational instability process requires $3 \mathrm{D}$ modeling of both the gas and stars embedded in a realistic galactic gravitational potential field.

Recently, a new class of high-resolution numerical galaxy formation simulations have arisen that begin to probe the density and resolution scales necessary to resolve star forming clouds. These models include a variety of simplifying assumptions, including an analytic stellar background potential, both with and without imposed spiral arms (Dobbs et al. 2006; Tasker \& Tan 2009; Tasker 2011; Smith et al. 2014; Dobbs et al. 2015; Tasker et al. 2015). Others resolve the gas to very high spatial resolution at the cost of capturing only a fraction of a galactic dynamical time, or of simulating a galaxy much smaller than the Milky Way (Bournaud et al. 2010; Renaud et al. 2013). Still others take a similar approach to ours (Agertz et al. 2009; Hopkins et al. 2012; Fujimoto et al. 2014a, 2014b; Behrendt et al. 2015), including runs based on the same AGORA (Kim et al. 2014) initial conditions that we use (Agertz et al. 2015).

In this paper we make use of simulations of the gas, stars, and dark matter in idealized isolated models of star forming disk galaxies. We focus on the spatial and mass scale similar to the Milky Way and study three different disks with varying gas fractions. We expand on previous work in a number of ways:

1. Using our high numerical resolution $-\Delta x \sim 20 \mathrm{pc}$ at the maximum refinement level, corresponding to a threshold density for star formation, $n_{\text {thresh }} \sim 50 \mathrm{~cm}^{-3}$-we are able to comfortably resolve the global properties of star forming regions in our disk models (although we do not resolve the internal properties of star forming clouds), allowing us to resolve an ISM with multiple distinct phases. 
2. Our simulations are run for several galactic dynamical times. This long time baseline allows the instability to reach a saturated, fully chaotic state. As we will show, the onset of violent gravitational instability in our disk models is accompanied by substantial transient ringing behavior characteristic of the smooth unstructured initial conditions, and we run for long enough for this initial state to be forgotten.

3. We perform a detailed modeling of the Toomre $Q$ parameter, taking into account contributions of the gas and stars, including both the thermal and kinetic gas pressure, and accounting for the nonzero thickness of the gas and stellar disks.

4. We measure the radial and temporal variation in the rate of radial mass transport at high spatial and temporal resolution, allowing us to track the detailed dynamics of the flow of matter in the disk and measure a mean flow of matter through the disk.

5. The initial conditions data, full simulation code, simulation outputs, and analysis code are publicly available. ${ }^{3}$

Our simulations are quite similar to those of Agertz et al. (2015). Their simulations are based on nearly identical initial conditions but were run with a factor of two higher spatial resolution than we use. On the other hand, we simulate galaxies over much longer baselines (600 Myr versus $140 \mathrm{Myr}$ ), which is essential to allow the gravitational instability to settle reach full nonlinear saturation and thereby to allow an accurate measurement of the rates of the typical mass transport rate due to gravitational instability. Agertz et al. (2015) do not consider the issue of transport in their study.

In addition, our simulations can be compared to the numerical experiments of Behrendt et al. (2015). They focused on the transition from the linear to nonlinear phase of gravitational instability in the context of an analytic treatment of the Toomre instability. We see similar morphology in our simulations - an initially smooth disk that breaks up into axissymmetric rings which then further segment and collapse into bound clumps. Their simulations were run with an isothermal equation of state and did not include a stellar disk and furthermore did not consider radial mass transport.

This paper is part of a series. In the companion paper (N. J. Goldbaum et al. 2015, in preparation) we perform simulations including stellar feedback, and compare these to the pure-gravity simulations presented here. A previously published paper in this series (Petit et al. 2015) makes use of one of the suite of simulations we describe here in order to study metal transport by gravitational instability, and thus we will not examine that topic further in this work.

The plan for the remainder of this paper is as follows. In Section 2 we describe the code, subgrid physics models, and initial conditions. In Section 3, we describe in general terms the time evolution of our simulations, particularly with respect to the dynamical evolution of the gravitational instability process. In Section 4 we quantitatively measure the effect of the gravitational instability via the time evolution of the $Q$ parameter, as well as the radial mass flux, measure the mean mass flux rate through our simulated disks, and discuss the velocity structure of the gaseous disk. Finally in Section 5 we review our findings and discuss implications for future work.

\footnotetext{
3 http://dx.doi.org/10.13012/J8F769GV
}

Table 1

Simulations

\begin{tabular}{|c|c|}
\hline \multicolumn{2}{|c|}{ Halo Parameters } \\
\hline$M_{200}$ & $1.1 \times 10^{12} M_{\odot}$ \\
\hline$V_{200}$ & $150 \mathrm{~km} \mathrm{~s}^{-1}$ \\
\hline$R_{200}$ & $206 \mathrm{kpc}$ \\
\hline$f_{c}$ & 10 \\
\hline$\lambda$ & 0.04 \\
\hline \multicolumn{2}{|c|}{ Disk Parameters } \\
\hline$M_{*}$ & $4.3 \times 10^{10} M_{\odot}$ \\
\hline$h_{R}$ & $3.4 \mathrm{kpc}$ \\
\hline$h_{z}$ & $0.34 \mathrm{kpc}$ \\
\hline \multicolumn{2}{|c|}{ Bulge Parameters } \\
\hline$M_{\mathrm{B}}$ & $4.3 \times 10^{10} M_{\odot}$ \\
\hline \multicolumn{2}{|c|}{ Resolution Parameters } \\
\hline$\Delta x$ & $20 \mathrm{pc}$ \\
\hline$n_{\text {thresh }}$ & $50 \mathrm{~cm}^{-3}$ \\
\hline$N_{\mathrm{H}}$ & $10^{7}$ \\
\hline$N_{\mathrm{D}}$ & $10^{7}$ \\
\hline$N_{\mathrm{B}}$ & $1.25 \times 10^{6}$ \\
\hline$m_{\mathrm{H}}$ & $1.3 \times 10^{5} M_{\odot}$ \\
\hline$m_{\mathrm{D}}$ & $3.4 \times 10^{3} M_{\odot}$ \\
\hline$m_{\mathrm{B}}$ & $3.4 \times 10^{3} M_{\odot}$ \\
\hline \multicolumn{2}{|c|}{ Gas Fraction } \\
\hline LGF & $10 \%$ \\
\hline Fiducial & $20 \%$ \\
\hline HGF & $40 \%$ \\
\hline
\end{tabular}

Note. All three simulations are initialized with the same halo, disk, bulge, and resolution parameters.

\section{SIMULATIONS}

This paper makes use of three simulations of isolated disk galaxies. All simulations were performed using the Enzo AMR hydrodynamics code (The Enzo Collaboration et al. 2014). These simulations include self-gravity, cooling, high-order shock-capturing hydrodynamics, and $\mathrm{N}$-body dynamics. The properties of the simulations are summarized in Table 1.

\subsection{Initial Conditions}

The initial conditions were generated for the AGORA project (Kim et al. 2014) using the makegalaxy code (Springel et al. 2005). Briefly, makegalaxy transforms an input halo mass $\left(M_{200}\right)$, stellar disk mass $\left(M_{*}\right)$, gas fraction $\left(f_{g}\right)$, halo spin parameter $(\lambda)$, and halo concentration parameter $\left(f_{c}\right)$ into particle initial conditions. These input parameters uniquely set the rotation curve, $V_{C}(R)$, and radial scale length, $h_{R}$, via the analytic theory of Mo et al. (1998).

The dark matter and stars are represented in the galaxy model using collisionless particles and are initialized by stochastically drawing from analytic distribution functions. The dark matter positions are initialized to follow a Hernquist (1990) distribution, which closely matches the more commonly used Navarro et al. (1996) fitting formula, but is more analytically tractable. The star particle positions are initialized 
following

$$
\rho_{*}(R, z)=\frac{M_{*}}{4 \pi h_{z} h_{R}^{2}} \operatorname{sech}^{2}\left(\frac{z}{2 h_{z}}\right) \exp \left(-\frac{R}{h_{R}}\right),
$$

where $M_{*}$ is the mass of the stellar disk, $h_{z}$ is the vertical scale height, $z$ is the vertical coordinate, and $R=\sqrt{x^{2}+y^{2}}$ is the cylindrical radial coordinate. We consider gas fractions $f_{g}=0.1,0.2$, and 0.4 , which we refer to as the low gas fraction (LGF), fiducial, and high gas fraction (HGF) runs, respectively (Table 1). Once the particle positions are computed, the velocities are populated using a distribution function that depends only on the local orbital energy $E$ and vertical component of the angular momentum $L_{z}$. The velocity distribution function is assumed to be axissymetric, making it strraightforward to locally solve the Jeans equation making use of the known density distribution from above (see Springel et al. (2005) for more details).

In addition to the stellar disk component, we also include a bulge of mass $M_{\mathrm{B}}$. The bulge is initialized following a Hernquist profile:

$$
\rho_{\mathrm{B}}(r)=\frac{M_{\mathrm{B}}}{2 \pi} \frac{b}{r\left(r+h_{\mathrm{B}}\right)^{3}},
$$

where $h_{\mathrm{B}}$ is a free parameter. The bulge-to-total mass ratio, $M_{\mathrm{B}} /\left(M_{\mathrm{B}}+M_{*}\right)$, is 0.1 in all three runs.

We include $N_{\mathrm{H}}=10^{7}$ halo particles, $N_{\mathrm{D}}=10^{7}$ particles in the stellar disk, and $N_{\mathrm{B}}=1.25 \times 10^{6}$ particles in the stellar bulge. All particles in each population have uniform masses. For the halo population, the mass of each particle is $m_{\mathrm{H}}=1.3 \times 10^{5} M_{\odot}$. For the bulge and disk population the particle masses are $m_{\mathrm{D}}=m_{\mathrm{B}}=3.4 \times 10^{3} M_{\odot}$.

Since makegalaxy produces initial conditions formatted for the gadget smoothed particle hydroynamics (SPH) code, special care must be taken to initialize the gas onto Enzo's AMR grid structure. Rather than interpolating from the initial conditions for the SPH particles, we instead initialize the gas density following an analytic density profile:

$$
\rho(R, z)=\frac{M_{\mathrm{G}}}{4 \pi h_{R}^{2} h_{z}} \exp \left(\frac{-R}{h_{R}}\right) \exp \left(\frac{-|z|}{h_{z}}\right),
$$

where $M_{\mathrm{G}}=f_{g} M_{*}$ is the mass of the gaseous disk. The gas velocities are initially axisymmetric, with the radial profile set according to the circular velocity curve written out by makegalaxy.

This departs somewhat from the procedure used by makegalaxy to generate SPH initial conditions, where the gas density distribution is assumed to be exponential in the radial direction but the vertical extent is determined via an iterative relaxation process to ensure the gas disk is initially in equilibrium. This relaxation process assumes that the gas pressure is moderated by a subgrid effective equation of state model, which we do not employ in this study. In practice, the gas in our simulations initially experiences a phase of violent collapse and relaxes into a quasi-equilbrium state after the gravitational instability has fully developed. This means the precise state of the gas in the initial conditions is not terribly important - the simulation "forgets" the gas initial conditions. Furthermore, this choice also allows us to make an apples-toapples comparison with future isolated galaxy simulations that make use of the public AGORA isolated galaxy initial conditions.

\subsection{N-body Dynamics, Hydrodynamics}

Rather than making use of an analytic dark matter and stellar potential (see, e.g., Dobbs et al. 2006; Tasker \& Tan 2009), we employ a live dark matter halo and stellar disk. This allows us to follow the active response of the stars and dark matter to the collapse of the gaseous disk. The particle dynamics are implemented in Enzo using a standard particle-mesh scheme (Hockney \& Eastwood 1988). Particle positions and velocities are updated according to the local gravitational acceleration using a drift-kick-drift scheme.

Hydrodynamics are captured using the piecewise parabolic method (PPM; Colella \& Woodward 1984). PPM hydrodynamics allows us to accurately capture strong shocks in a few computational zones while also maintaining second-order spatial accuracy. While the PPM method is formally secondorder accurate in space and time, Enzo's adaptive timestepping scheme is only first-order accurate, so these calculations are only first order accurate in time. In addition, after experiencing intermittent instability using second-order interpolation, we opted to degrade to first-order interpolation at AMR level boundaries. This means that we are formally first-order accurate in space, although these inaccuracies should only show up at level boundaries.

Since the $\sim 200 \mathrm{~km} \mathrm{~s}^{-1}$ circular velocity of the galaxy necessitates strongly supersonic flows in the galactic disk, we make use of the dual energy formalism implemented in the Enzo code (Bryan et al. 1995). In standard PPM hydrodynamics, the internal energy is not tracked-instead it is derived from the total energy and velocity. When the kinetic energy is much larger than the internal energy, this can lead to spurious temperature fluctuations due to floating point roundoff error. To avoid this, Enzo tracks a separate internal energy field that in effect provides extended precision to the total energy field. This allows us to safely resolve the thermal physics of the gas in a global simulation of a disk galaxy without worrying about spurious temperature fluctutions due to advection errors.

\subsection{Initial Grid Structure and Refinement Criteria}

Our simulations make full use of the AMR capabilities of the Enzo code. Our refinement strategy is to focus computational effort on the highest refinement level, while still adequately resolving the dark matter halo so our estimate of the gravitational potential in the neighborhood of the galactic disk is accurate. Since the dark matter halo is spatially extended compared to the disk, with the furthest dark matter particles living $500 \mathrm{kpc}$ away from the center of the galaxy, this necessitates a hierarchically nested AMR structure.

To fully encompass the dark matter halo, we employ a cubic simulation box with a width of $1.3 \mathrm{Mpc}$, and resolve the root grid with $64^{3}$ cells. In addition to the static root grid, we impose 5 additional levels of statically refined regions, enclosing volumes that are successively smaller by a factor of 8 .

In addition to the static refinement, we allow for an additional five levels of adaptive refinement. To keep the particles properly resolved at all times, we refine a cell if the total mass in particles within the cell exceeds $1.7 \times 10^{6} M_{\odot}$, or approximately 10 halo particles. This choice produces nested 
grid hierarchies in the region of the simulation dominated by the dark matter halo where the grid hierarchy is determined adaptively.

To keep the gaseous disk resolved at all times, we refine a cell if the total mass in gas within the cell exceeds $2.2 \times 10^{4} M_{\odot}$. Finally, to avoid artificial fragmentation, we ensure the Jeans length, $\lambda_{\mathrm{J}}=\sqrt{\left(\pi c_{s}^{2}\right) /(G \rho)}$, is locally resolved at all times by at least 32 cells, comfortably satisfying the Truelove et al. (1998) criterion. To keep the Jeans length resolved after collapse has reached the maximum refinement level, we employ a pressure floor such that the Jeans length is resolved by at least four cells on the maximum refinement level. To avoid contaminating the hydrodynamics in situations where we can use more resolution, the pressure floor is only employed for cells on the maximum refinement level. The pressure floor is implemented as a source of "extra" pressure above the thermal pressure-an improvement over the Jeans stabilization routines in the current stable version of Enzo where the pressure floor is implemented by increasing the temperature. The new pressure floor implementation will be included in the next stable release of Enzo.

The Enzo AMR hierarchy is fully adaptive in space and time, so regions that no longer satisfy the refinement criteria described above will no longer be refined. We do not include an explicit de-refinement criteria, instead allowing the refinement criteria described above to fully control the AMR hierarchy.

The particle refinement criteria we use are not as stringent as the gas refinement criteria. If the gas were not present in the simulation, the star refinement criteria alone would cause the stellar disk to be refine to AMR level $7(160 \mathrm{pc})$ resolution in the outskirts of the disk and AMR level 8 ( 80 pc resolution in the inner disk and bulge. Since the combined contribution of the gas and stars determine the final resolution of the stars, in general the stellar disk is resolved to $20-40$ pc resolution. In particular, the resolution is higher near dense clumps and filamentary structure in the gas. Early in the simulation, before the gas has had a chance to be consumed by star formation, the resolution is also generally higher.

Near the beginning of all three simulations, there are $3 \times 10^{7}$ computational zones in total, with about $1.5 \times 10^{7}$ zones on the maximum refinement level. Toward the end of the fiducial and high gas fraction simulations, after much of the gas disk has been consumed the number of zones on the maximum refinement level decreases to $\sim 5 \times 10^{6}$ zones. We attribute this decline to the collapse of gas into dense clumps and the consumption of gas by star formation, leaving much of the volume occupied by relatively rarefied gas.

\subsection{Star Formation}

Since the gaseous disks in our simulations are unstable to fragmentation and collapse, it is necessary to include a subgrid model that converts dense, collapsing gas into newly formed star particles. Rather than using the built-in "standard" star formation prescription in the En zo code (Cen \& Ostriker 1992; The Enzo Collaboration et al. 2014), which is tuned for lowerresolution cosmological simulations, we make use of a new star formation prescription. To ease comparison with future papers using the AGORA initial conditions, the prescription is based on the suggested star formation model for the AGORA project (Kim et al. 2014).
Briefly, the model assumes that the star formation rate density in any cell is a function only of the gas density in that cell, according to the following formula:

$$
\frac{d \rho_{*}}{d t}= \begin{cases}f_{*} \frac{\rho}{t_{\mathrm{ff}}} & : \rho>\rho_{\text {thresh }} \\ 0 & : \rho \leqslant \rho_{\text {thresh }},\end{cases}
$$

where $f_{*}$ is the star formation efficiency, $t_{\mathrm{ff}}=\sqrt{3 \pi / 32 G \rho}$ is the local dynamical time, and $\rho_{\text {thresh }}=\mu m_{\mathrm{h}} n_{\text {thresh }}$ is the threshold density for star formation. Here $\mu$ is the fixed mean molecular weight, $m_{\mathrm{h}}$ is the mass of a hydrogen atom, and $n_{\text {thresh }}=50 \mathrm{~cm}^{-3}$ is the threshold number density. Note that since we do not advect species fractions for hydrogen and helium ionization states, $\mu=1.4$ is constant over the full simulation box. The value we choose is typical of the bulk of the atomic ISM. In all of our simulations, we use $f_{*}=1 \%$, in accordance with the observed low star formation efficiency universally observed in star forming regions at a range of density and size scales (Krumholz \& Tan 2007; Krumholz et al. 2012).

The star formation threshold is chosen such that the Jeans length for gas on the maximum refinement level remains resolved by at least four cells until the refinement reaches the maximum level, assuming a temperature floor of $100 \mathrm{~K}$, approximately the minimum temperature we see in our simulations. Once gas reaches the maximum allowed refinement level, the gas is no longer well resolved, and we allow the gas to convert itself into stars and apply a pressure floor to prevent artificial fragmentation. Since we only barely resolve the typical densities for the formation of molecular gas, we do not employ a subgrid model to track the molecular gas fraction, instead assuming that gas above the threshold density is fully molecular. This follows Guedes et al. (2011), who found that the precise value chosen for $\rho_{\text {thresh }}$ was not important for determining the star forming properties of a disk galaxy in a cosmological zoom-in simulation, so long as the threshold density is high enough that star formation occurs primarily in dense clumps of gas rather than in the bulk of the ISM.

Practically speaking, Equation (4) cannot be solved by spawning star particles at each timestep for all cells with densities above the threshold density. Given a typical timestep of 1000 years, and assuming a cell containing gas at the threshold density, we would expect to be spawning star particles with masses of only $0.01 M_{\odot}$. Rather than spawning star particles in every cell that exceeds the density threshold, we instead impose a minimum star particle mass, and form stars stochastically. In our scheme, a cell will form a star particle of mass $m_{\mathrm{sf}}=300 M_{\odot}$ with probability

$$
P_{*}= \begin{cases}f_{*} \frac{\rho \Delta x^{3}}{m_{\mathrm{sf}}} \frac{d t}{t_{\mathrm{ff}}} & : \rho>\rho_{\text {thresh }} \\ 0 & : \rho \leqslant \rho_{\text {thresh }},\end{cases}
$$

where $d t$ is the simulation timestep on the maximum refinement level. By stochastically creating according to Equation (5), the time-averaged value of the star formation rate in each cell is still given by Equation (4), while creating a reasonable number of particles.

In Figure 1, we compare the star formation rate inferred from the ages of star particles present at the end of each simulation with the expected star formation rate measured by evaluating 


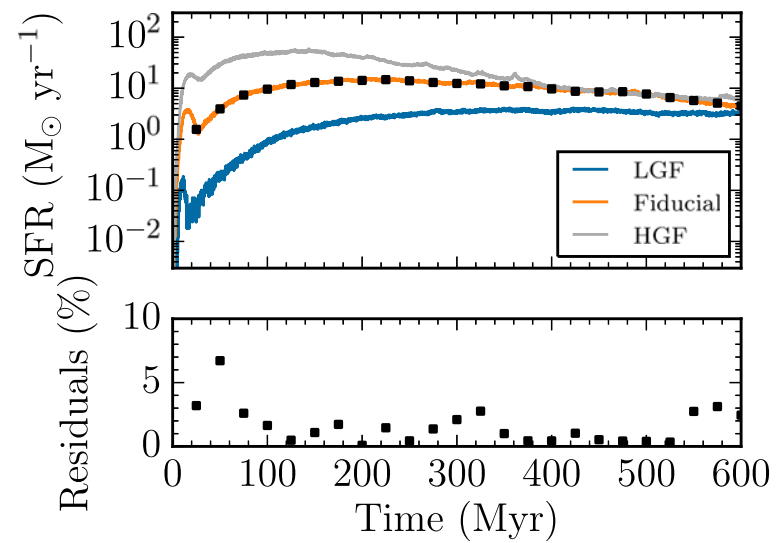

Figure 1. Observed (solid lines) star formation rate as a function of time for all three simulations. The black squares were calculated by directly measuring the expected star formation rate in the fiducial simulation according to Equation (4). The blue, orange, and gray lines were calculated by binning the star particles that are dynamically formed in the simulation by their formation times. In the bottom panel we plot the residual between the black squared and orange line in the top panel. We find good agreement between the predicted and measured star formation rate.

Equation (4) at $25 \mathrm{Myr}$ intervals. We see that there is good agreement between the measured and predicted star formation rate, with a maximum deviation of $\sim 7 \%$. The implementation of this star formation algorithm has been made publicly available and will included in the next stable release of Enzo.

\subsection{Heating and Cooling}

To model the thermal physics of the gas in our simulations, we make use of the Grackle cooling library ${ }^{4}$ (Kim et al. 2014; The Enzo Collaboration et al. 2014). The Grackle cooling and heating routines were adapted from the chemical and thermal physics implementation included in the Enzo code. Relative to Enzo's implementation of thermal physics, Grackle adds novel capabilities that we make use of in this study. In addition, this choice eases comparison with future simulations performed in other codes as well as simulations performed for the AGORA comparison.

Grackle includes a primordial cooling routine based on tabulated cooling rates as a function of density and temperature, avoiding the need to evolve separate fields for each hydrogen and helium ionization state. In addition to cooling by primordial species, we also include cooling due to metal line emission. These cooling rates are inferred from tabulated rates output by the CLOUDY code.

The disk is initialized to include a metal color field that follows the initial gas distribution. All gas zones in the disk are initialized with a solar metal fraction. The metal field is passively advected along with the gas. We include a dynamical metal color rather than assuming a fixed metal fraction in anticipation of modeling the production of metals in simulations with supernova feedback.

To properly model the thermal physics in a Milky Way-like ISM, we include a prescription for heating due to electrons released from dust grains by the photoelectric effect. This is implemented as a constant heating rate of $8.5 \times 10^{-26} \mathrm{erg} \mathrm{s}^{-1}$ per Hydrogen atom applied uniformly throughout the simulation box for gas below $10^{4.3} \mathrm{~K}$ (Tasker 2011). This rate is

\footnotetext{
4 https://grackle.readthedocs.org
}

chosen to match the expected heating rate assuming a UV background consistent with the solar neighborhood value (Draine 2011).

\subsection{Analysis}

In order to analyze our simulations, we construct an extensive post-processing pipeline using a combination of the yt toolkit (Turk et al. 2011) and a set of custom python analysis scripts that we have made publicly available on Bitbucket. ${ }^{5}$ Details of the analysis pipeline, and how we reconstruct various quantities of interest from the raw simulation outputs, are described in Appendix A. In particular, Appendix A gives our formal definitions of quantities that are non-trivial to calculate, including velocity dispersions (Appendix A.4), effective sound speeds (Appendix A.5), and Toomre $Q$ parameters (Appendix A.6).

\section{QUALITATIVE OUTCOME}

We present snapshots of the fiducial simulation at four times in Figure 2 and snapshots of all three simulations at a fixed time in Figure 3. Each figure displays the gas and stellar surface density and effective sound speed as well as the combined Toomre $Q$ parameter.

The dynamics of all three simulations are similar. The initially smooth gaseous disk quickly cools from the initial temperature of $10^{4} \mathrm{~K}$ to $200-300 \mathrm{~K}$. The initially thermally supported disk proceeds to collapse from vertically over the course of the next 20-50 Myr. Denser regions in the center of the disk collapse first, followed by less dense regions further out in the disk, with the precise collapse time determined by the initial gas surface density at any given radius. Once the gas has collapsed in the vertical direction, the disk remains very thin for the rest of the simulation, with the bulk of the gas only one or two cells (20-40 pc) away from the midplane. Our disks are thus not well resolved in the vertical direction.

Once the gas has collapsed vertically, a combination of shear and self-gravity shepherds the gas into filaments which in turn collapse into isolated gravitationally bound clouds. Since these simulations do not include feedback, these clouds survive more or less permanently, only disappearing if they exhaust their gas supply by converting gas into stars, or merge with one another. As the gas in the gravitationally bound clouds is converted into stars, massive star clusters form inside the clouds. Once the initial period of collapse and fragmentation has passed, both the gas and stars spontaneously align into clear spiral arms. Later, as an increasing fraction of both the gas and stars collects inside the gravitationally bound clumps, the spiral structure becomes less clearly defined. These long-lived dense clouds are very similar to those seen by Hopkins et al. (2012) in their simulations with no feedback.

Gas in the innermost regions is stabilized against fragmentation by the presence of the bulge. Rather than forming gravitationally bound clouds, the gas in the bulge quickly stabilizes into a thin disk. Since the disk is above our density threshold for star formation, the central disk disappears as it converts itself into newly formed stars. We caution that star formation in the bulge might be an artifact of our $20 \mathrm{pc}$ resolution, since we do not see any fragmentation there and the

\footnotetext{
5 https://bitbucket.org/ngoldbaum/galaxy_analysis
} 


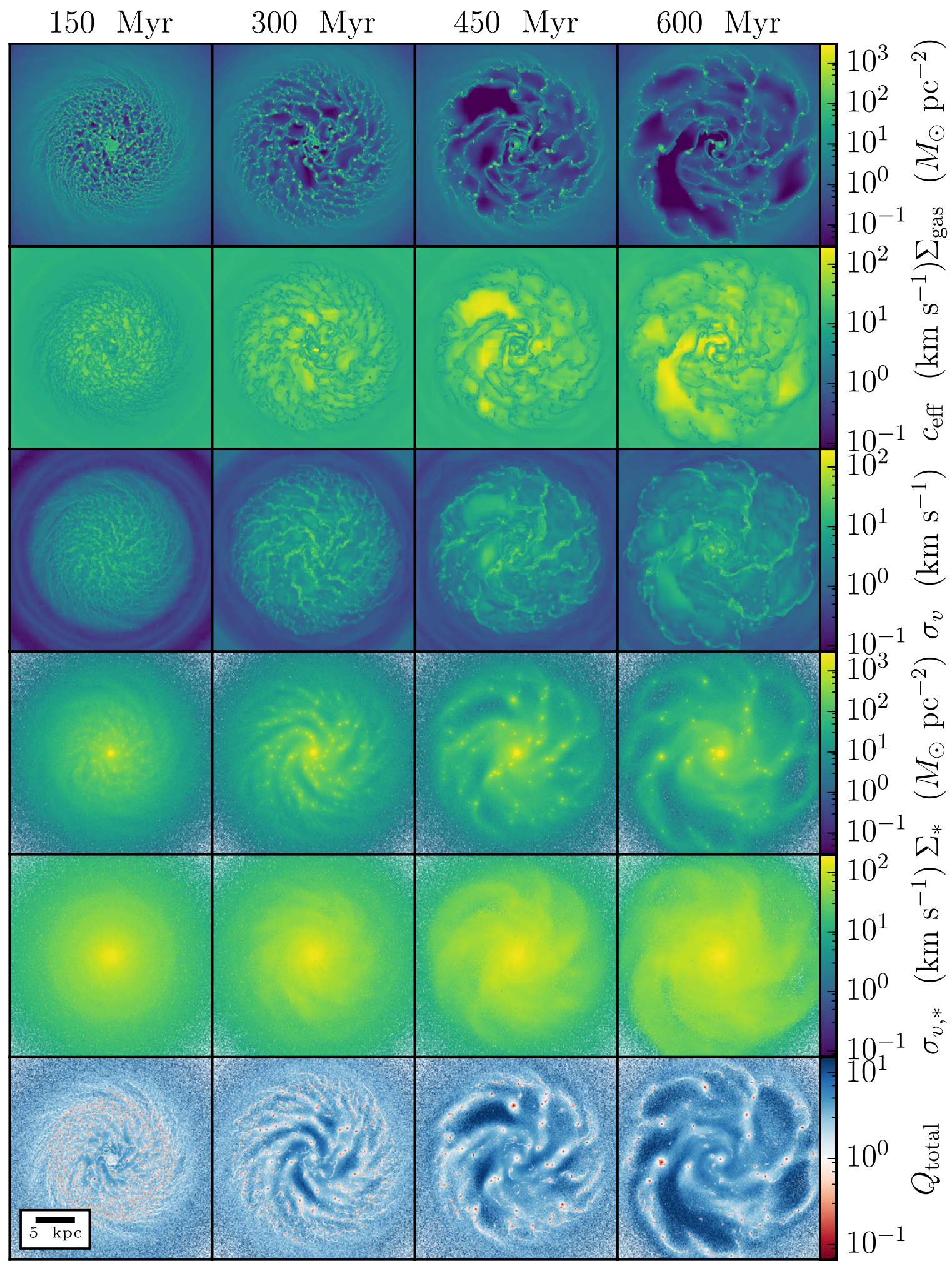

Figure 2. Time evolution of the gas and stars in the fiducial simulation. The quantities shown are, from top to bottom, gas surface density, effective sound speed of the gas (including thermal and turbulent contributions), gas velocity dispersion, stellar surface density, stellar velocity dispersion, and total (gas plus stars) Toomre $Q$; formal definitions for all quantities are given in Appendix A. The simulation time for each column is indicated at top and the spatial scale is indicated by the scale bar at the bottom left. Each panel displays a region $25 \mathrm{kpc}$ across centered on the galaxy.

bulk of the gas in the bulge is at or above our star formation threshold density.

The combination of gravitational collapse and shear induces significant turbulent motion in the gas, with typical velocity dispersions of $10-20 \mathrm{~km} \mathrm{~s}^{-1}$ in the portion of the disk that participates in gravitational instability $(R \lesssim 15 \mathrm{kpc})$. The hot, low-density gas in the interarm regions exhibits slightly higher turbulent velocity dispersions than the dense gas, but with 


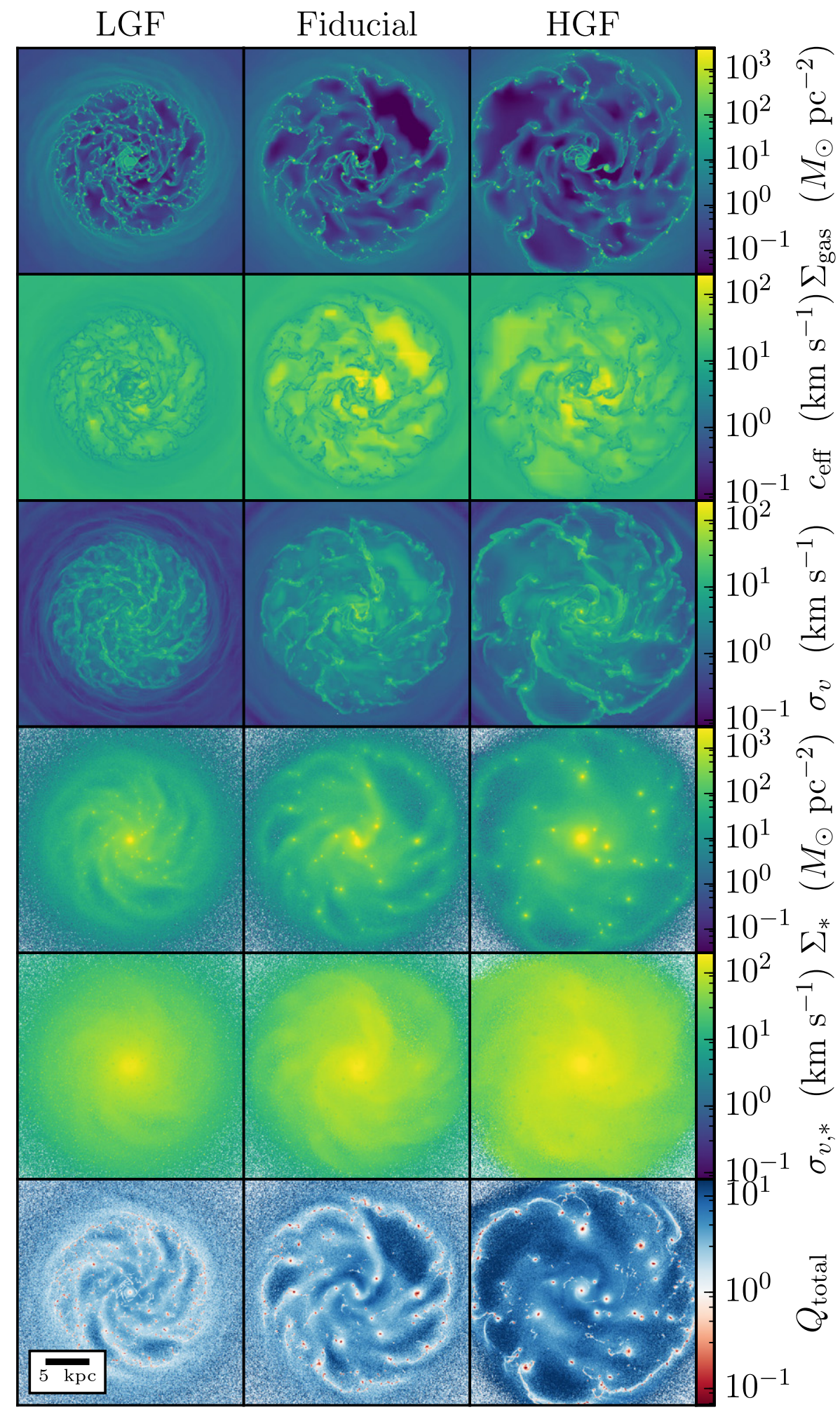

Figure 3. Same as Figure 2, but for each of the three different simulations at $T=500 \mathrm{Myr}$.

much lower typical Mach numbers. In the interarm region, the effective sound speed is typically $100-200 \mathrm{~km} \mathrm{~s}^{-1}$ and is dominated by the thermal sound speed, while in the dense gas the turbulent velocity dispersion dominates the effective sound speed. Thermal sound speeds are high in the interarm regions because these are heated by spiral shocks, and their low densities leave them unable to cool effectively afterwards. In contrast, the denser arm gas quickly cools back to its equilibrium temperature. The stellar orbits do not experience significant heating or dissipation, retaining the $\sim 40 \mathrm{~km} \mathrm{~s}^{-1}$ velocity dispersion present in the initial conditions. We also note that the kinetic energy content of the interarm gas is negligible compared to the gas in dense clumps, and does not contribute significantly to the kinetic energy budget of the disk. 
The Toomre $Q$ parameter tends to increase in time. In our initial conditions for all three simulations, $Q \sim 1$ throughout the disk. As soon as the gas is able to cool and begins to collapse, $Q \ll 1$. As collapse proceeds, the typical surface density decreases and the degree of turbulence increases, leading to $Q$ steadily increasing in time. Regions in which gravitationally bound clumps develop consistently exhibit $Q \ll 1$, even at late stages, but, as we show below, azimuthal averages of $Q$ are always greater than one. The locally low values of $Q$ within the bound clumps can mostly be attributed to very high gas and stellar surface densities in these regions. We do not see any significant increase in the stellar velocity dispersion in these regions, and we see only a modest increase in the gas effective sound speed. See Section 4.1 below for more discussion of how $Q$ evolves with time.

The stellar and gas dynamics in all three simulations are similar, but the detailed dynamics do vary somewhat as a function of initial gas fraction. Increasing the gas fraction leads to quicker collapse. Decreasing the gas fraction produces a slower collapse in which the gas spends a longer amount of time in filaments rather than dense clumps. Spiral arms are more prominent in the LGF simulation, presumably because they do not have enough time to be disrupted by the formation of gravitationally bound clouds and accompanying star clusters. The star forming portion of the disk is also more compact, since regions at the outskirts of the disk are no longer dense enough to cool and collapse.

The degree of gravitational instability displays a weak dependence on the gas fraction. Since the high gas fraction run is able to process a much larger fraction of the gas present in the initial conditions, it reaches a higher typical $Q_{\text {total }}$ value due to the lower typical gas surface densities in the depleted gas disk. The LGF run exhibits less variation, with $Q_{\text {total }} \sim 1-3$ throughout the disk, though this may merely reflect that $Q_{\text {total }}$ rises more slowly with smaller initial gas fraction, and that we have run only for a few orbits. In any case, in all three cases, the disks are formally stable according to classical Toomre analysis, with $Q_{\text {total }} \gtrsim 1$. We consider this state to be the final, saturated result of gravitational instability unrestrained by any form of feedback. We make the notion of what constitutes such a steady state in our simulations more quantitative in Section 4.2, where we discuss mass transport through the disk.

A robust feature of all of our simulations is that gravitationally bound clouds form and then are unable to be destroyed by any mechanism besides gas exhaustion. This leads to the formation of large star clusters composed of stars that formed dynamically in the simulations. These star clusters in turn become a significant contribution to the mass distribution in the midplane, creating substantial streaming motions and departures from a smooth axisymmetric rotation curve. In the companion paper (N. J. Goldbaum et al. 2015, in preparation) we show that feedback capable of disrupting these complexes is necessary to form realistic smooth disks and prevent the formation of unrealistically massive star clusters.

\section{RESULTS}

\subsection{Gravitational Instability}

Since our simulated galaxies do not include a prescription for star formation feedback, the primary driver for the dynamical evolution of our model galaxies is gravitational instability. While the initial conditions for our simulations are formally

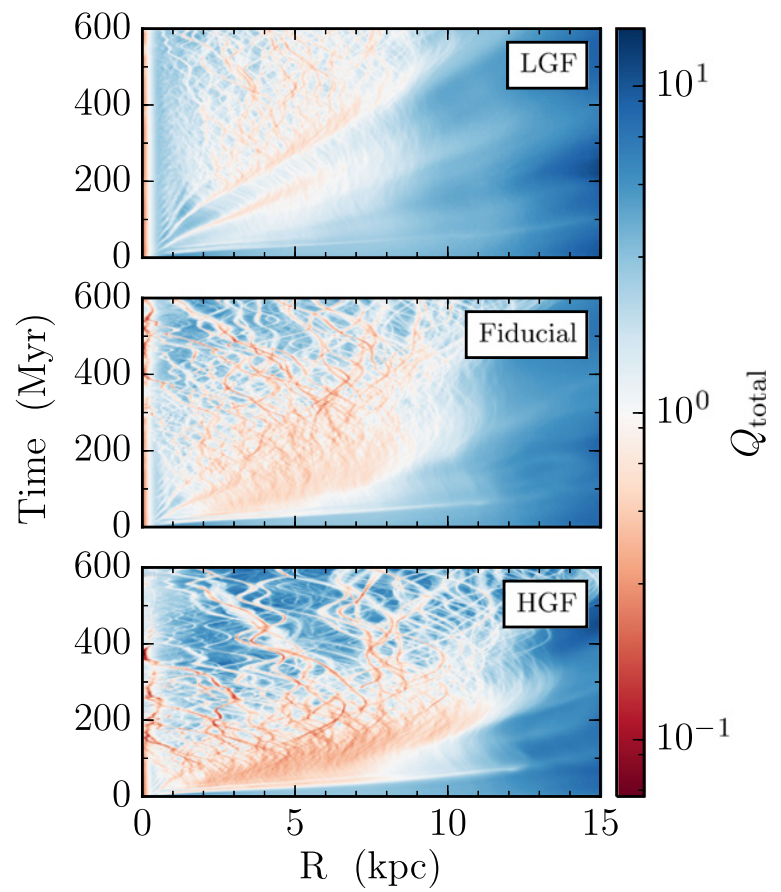

Figure 4. Time evolution of the azimuthally averaged Toomre $Q$ parameter in each of our simulations (see Equation (22)). This estimate includes the combined contribution of both the gas and stars. The color scale is chosen such that regions that are gravitationally unstable are colored red, regions that are marginally stable are colored white, and regions that are stable are colored blue.

stable, cooling allows the gas to quickly lose hydrostatic support, leading to catastrophic collapse in the vertical direction. In addition, our shearing self-gravitating disks are susceptible to the Toomre (1964) instability.

This story can be inferred by inspecting Figure 4, where we plot the time evolution of the azimuthal average of $Q_{\text {total }}$. The initially stable disk quickly becomes unstable (e.g., the regions that show up in red in the bottom half of each subplot). The instability leads to a radially expanding wave of collapsing gas. Soon after, the gas collects in gravitationally bound clumps, which proceed to migrate through the disk, both radially inward and outward. The regions inside the gravitationally bound clouds are formally unstable according to a local Toomre analysis due to their very high surface densities. The interclump regions reach relatively high values of $Q_{\text {total }}$ so these regions are formally stable to collapse.

This process plays out in all three simulations, albeit with varying collapse speeds and degrees of violence. The high gas fraction case initially develops filaments, but by $\sim 200 \mathrm{Myr}$ of evolution these have broken up into giant clumps everywhere in the disk. As we lower the gas fraction, the transition to the clump-dominated phase takes longer, and the clumps themselves become smaller. Nonetheless, all three simulations reach similar clump-dominated regimes, which appears to represent the fully saturated state of the gravitational instability.

We can see what is driving the evolution of $Q_{\text {total }}$ by inspecting Figure 5. We show the evolution in $Q_{\text {total }}$ (top row) along with the quantities that determine $Q_{\text {total }}$ : the gas and stellar surface density and effective sound speed, as well as the epicyclic frequency. We see that both the epicyclic frequency and the stellar surface density and velocity dispersion show little variation over the course of the simulations. 

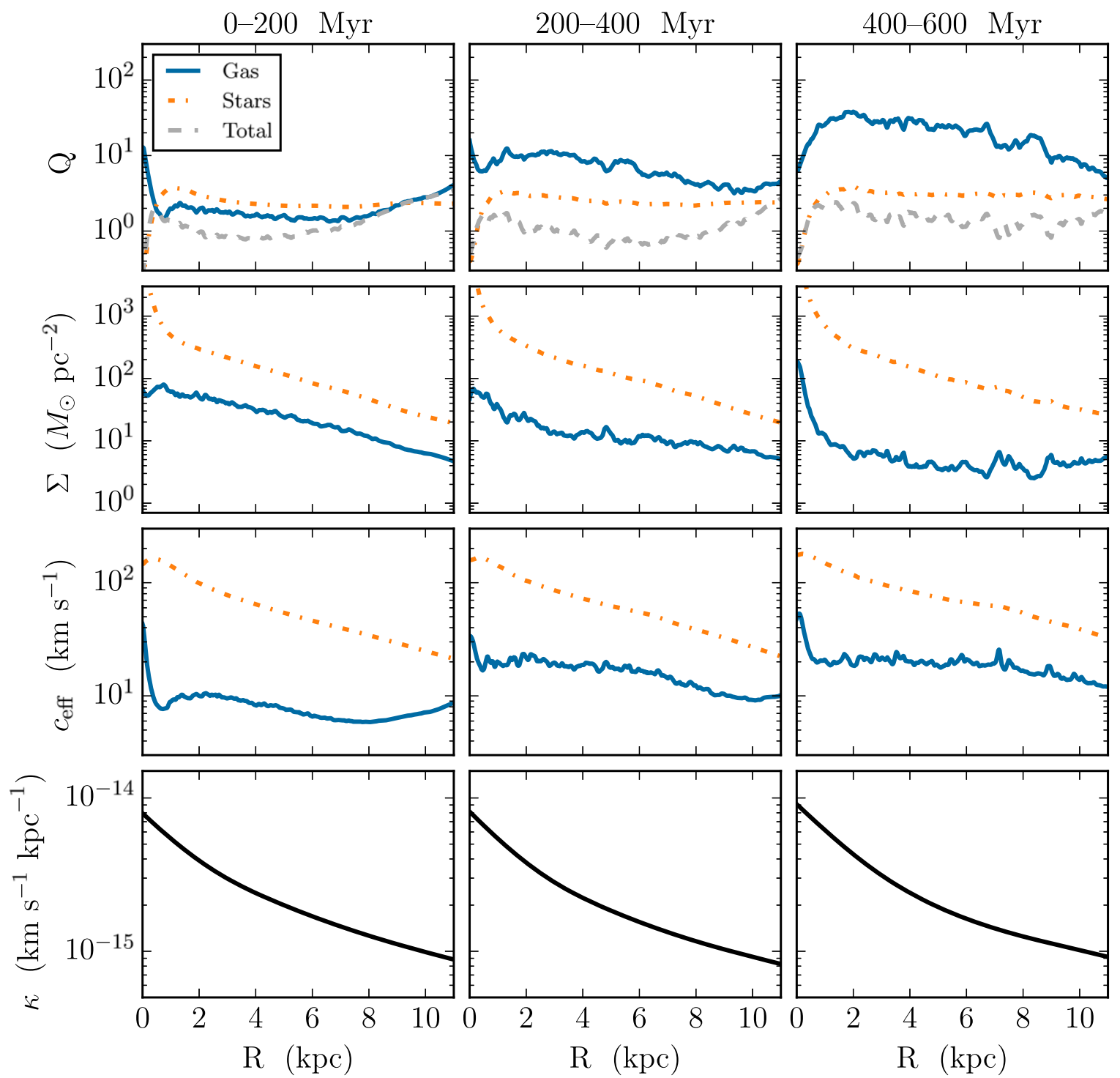

Figure 5. Time averages of the Toomre $Q$ parameter (top row), the surface density (second row), and effective sound speed (third row) for the gas and stars averaged over three different periods (indicated at top) in the fiducial simulation. The bottom row shows the local epicyclic frequency $\kappa$, which does not vary significantly over the course of the simulation. The evolution in $Q_{\text {total }}$ is primarily driven the evolution in $Q_{\text {gas }}$, which in turn is driven by depletion in the gas supply and and increase in the gas velocity dispersion.

On the other hand, the gas surface density and effective sound speed show significant variation. The effective sound speed tends to increase in time and the gas surface density tends to decrease. Both of these effects drive a secular increase in $Q_{\text {gas }}$ over the course of the simulation. In turn, this increase in $Q_{\text {gas }}$ leads to the secular increase in $Q_{\text {total }}$ Eventually, once the gas supply is exhausted, $Q_{\text {gas }} \gg 1$, so $Q_{\text {total }}$ approaches $Q_{*}$.

\subsection{Mass Transport}

Here we examine the radial flow of material in the disks of our simulated galaxy models. We measure if there is any net flow of material either from the galactic center outward or from the outskirts inward. In addition, we examine the detailed radial and time dependence in the gas mass flux.

In Figure 6, we present our measurements of the gas mass flux as a function of radius and time. All three simulations exhibit similar overall behavior. Initially, the models exhibit significant ringing as the disks initially collapse and settle down. In this stage the mass flux is dominated by rings of material experiencing alternating bands of inward and outward flow. After the initial collapse phase, the gas collects in gravitationally bound clouds. For the rest of the simulation, the mass flux rate is primarily determined by the inward and outward flow of the gravitationally bound clumps. As spiral arms develop, the gravitational potential in the disk begins to develop non-axisymmetric components that tend to drive the gas clumps both radially inward and outward.

Along with the detailed variation in the mass flux, we would also like to know if there is any net mass flux once the disk has settled down into a quasi-equilibrium state. To answer this question, we make use of the time averaging algorithm described in Appendix A.9. In this way we only consider the mass fluxes measured above the blue lines in Figure 6, which delineate the approximate time at which the disk has settled into a steady state.

This averaging procedure results in the time-averaged massflux measurements in the top panel of Figure 7. For each 


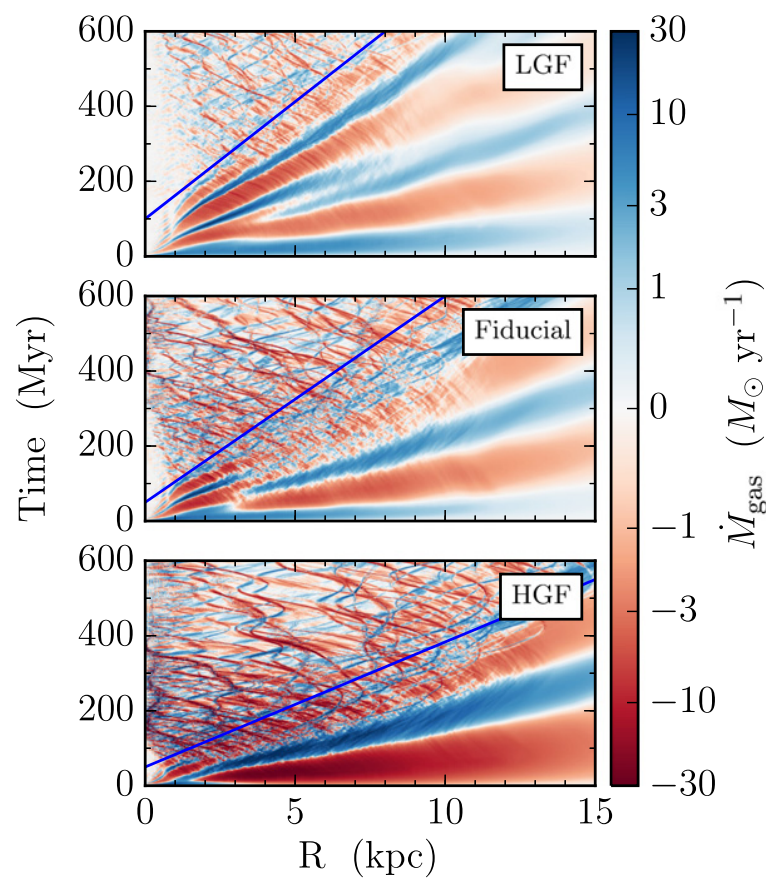

Figure 6. Gas mass flux as a function of radius and time for each of our simulations. We define $\dot{M}_{\text {gas }}$ such that positive values correspond to outward radial flow while negative values correspond to inward radial flow. The blue line indicates where we expect the disk has fully transitioned to an equilibrium condition, "forgetting" about the initial transients. Data below the blue line is excluded from the time averages plotted in Figure 7.
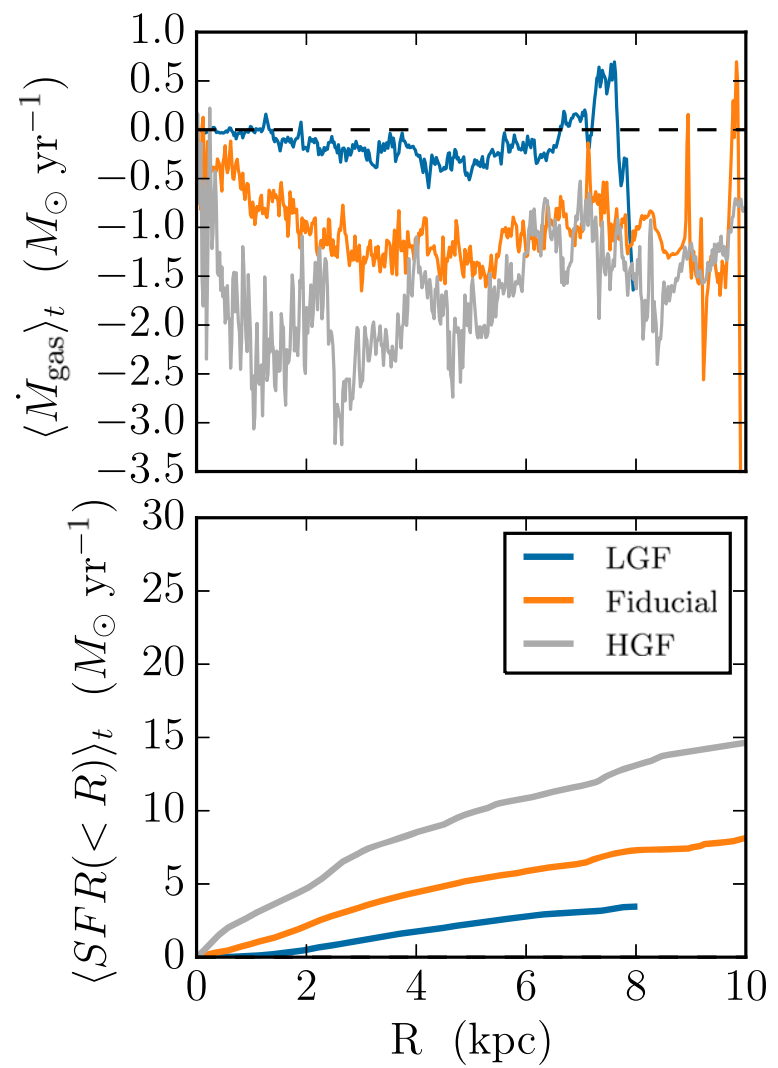

Figure 7. Time averaged mass flux (top panel) and radially cumulative star formation rate (bottom panel) as a function of galactocentric radius for all three simulations. For the top panel, positive values correspond to outward radial flow while negative values correspond to inward radial flow. In all cases there is a significant net inward flow of material toward the galactic center in the inner galaxy. simulation, we plot the time-averaged mass flux as a function of radius. We find that there is a net inward flux of gas at most radii for all three model galaxies. The flux scales very roughly with the gas fraction, with a typical mass flux of $-0.3,-1.0$, and $-2.0 M_{\odot} \mathrm{yr}^{-1}$ mass flux in the LGF, fiducial, and HGF cases, respectively.

Although the flow is inward at most radii in the disk, we note that the outer edges of the disks instead show an outward mass flux. (In Figure 7, this feature is outside the plotted region for the HGF case, but is visible for the Fiducial and LGF cases.) Comparing with Figure 4, we see that this outward mass flux appears roughly at the boundary between the gravitationally unstable region and the still-stable outer region of the disk. Such a reversal of the mass flow direction at the transition between the stable and unstable regions of a gravitationally unstable disk is consistent with the predictions of Forbes et al. (2014a). However, we caution that these outer regions have been averaged over for the shortest times, and so the exact value of the typical outward flux is uncertain.

We might expect that the inward flow of gas is sufficient to supply the star formation in the inner regions of our model galaxies. To see if this is the case, we plot the time-average of the radially accumulated star formation rate in the bottom panel of Figure 7. The mass flux in the top panel is sufficient to supply the star formation in the bottom panel only if the mass flux is greater than the cumulative star formation rate at any given radius. Due to the very high star formation rates in these simulations with no feedback (c.f. Figure 1), we find that the inward flow of gas is insufficient to fuel the star formation rate in these simulations.

However, we note that this is a result of the unphysically high star formation rates that our simulations exhibit due to the lack of star formation feedback. If we instead consider the star formation rate that would be expected given the gas surface density distribution combined with observed star formation rates (e.g., Leroy et al. 2013), we reach the opposite conclusion: our inflow rates are sufficient to fuel star formation at observed levels. Indeed, our fiducial, Milky Way-like simulation produces mass transport at a rate of $\sim 1 M_{\odot} \mathrm{yr}^{-1}$, which is roughly the observed star formation rate in the Milky Way (e.g., Chomiuk \& Povich 2011). The question of whether inflows and star formation can be matched simultaneously in a simulation including feedback we defer to the companion paper.

\subsection{Gas Velocity Structure}

Here we examine the detailed gas velocity structure in our simulated galaxies. We are particularly interested in the radial dependence in the effective sound speed, the anisotropy in the velocity dispersion, and the relative contribution of turbulent motions and thermal sound speed to the effective sound speed. In Figure 8 we present the time evolution of the azimuthally averaged effective sound speed in each of our simulations. In all three simulations, gravitational instability alone is able to drive substantial turbulent velocity dispersions in the inner disk. The outer, gravitationally stable disk is characterized by much lower velocity dispersions. We also note that the typical velocity dispersion scales with the initial gas fraction. This may be interesting for studies of high-redshift gas disks, which are known to exhibit substantial turbulent velocity dispersions.

To obtain a more complete picture of the velocity structure in our simulations, we look in more detail at the time-averaged 


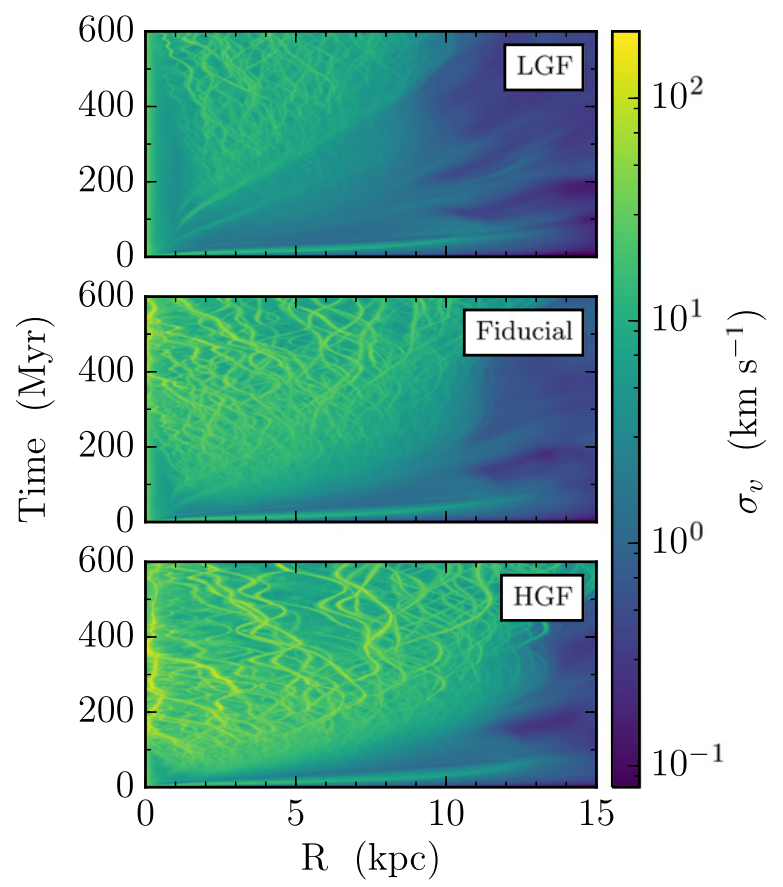

Figure 8. Time evolution of the azimuthally averaged velocity dispersion for the gas in each of our simulations. Gravitational instability alone is sufficient to drive substantial $\left(>10 \mathrm{~km} \mathrm{~s}^{-1}\right)$ velocity dispersions in all three simulations.

velocity structure in the fiducial simulation. We perform the same sort of time averages described in Appendix A.9, focusing on the velocity dispersion, sound speed, effective sound speed, and velocity anisotropy.

We present the results of this time averaging in Figure 9. In the top panel, we plot the average effective sound speed as a function of radius, along with the $1 \sigma$ dispersion in the effective sound speed at any given radius. The effective speed is typically of order $\sim 20 \mathrm{~km} \mathrm{~s}^{-1}$, with a gradual radial decline comparable to what is observed in $\mathrm{H}$ I maps of nearby galactic disks (see the discussion and references in Section 1.1). This is perhaps surprising given that feedback processes are often invoked as an energy source for turbulence. Instead, we find generically that gravitational instability alone is sufficient to drive substantial turbulent velocity dispersions, more than enough to match observed gas velocity dispersions in resolved observations of galactic gas kinematics.

Since the averages used in these profiles are mass weighted, the hot low-density interarm regions do not contribute significantly. As can be seen by inspecting Figure 2, the interarm gas has sound speeds of order $100 \mathrm{~km} \mathrm{~s}^{-1}$, but as we show in Figure 9, a typical gas parcel (in a mass-weighted sense) has an effective sound speed of order $20 \mathrm{~km} \mathrm{~s}^{-1}$. We make use of mass-weighted averages so that the measured effective sound speed profiles are representative of the kinetic energy content of the gaseous disk.

Our galaxy models look quite different from real galaxies when we look at the anisotropy in the velocity dispersion components. To investigate this, in the middle panel of Figure 9 we present the time-averaged, azimuthally averaged ratio of the vertical velocity dispersion to the in-plane velocity dispersion in the fiducial galaxy model, $\sqrt{2} \sigma_{v, z} / \sigma_{v, d}$. We include the factor of $\sqrt{2}$ in the numerator so that a value of unity would correspond to a flow field with isotropic turbulence, in which the dispersion in
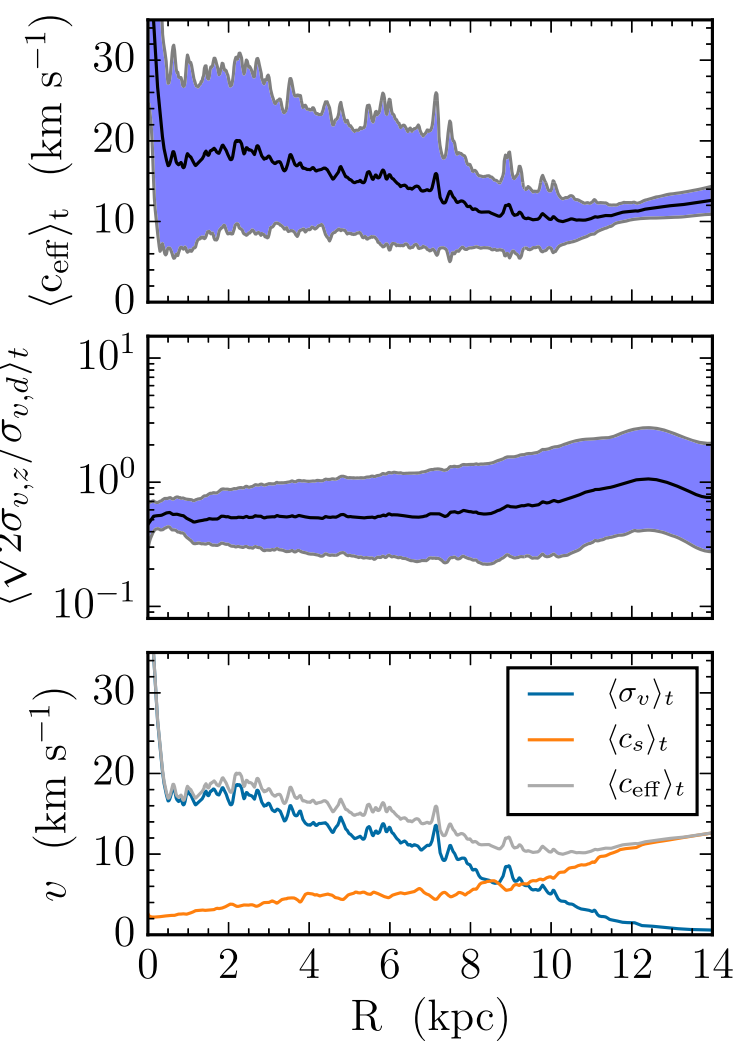

Figure 9. Time-averaged effective sound speed (top and bottom panel) and velocity dispersion anisotropy (middle panel) for the gas in our fiducial simulation. Shaded blue regions indicate the $1 \sigma$ scatter at a fixed radius. In the bottom panel, we show the contribution to the effective sound speed due to the velocity dispersion and sound speed. In the inner disk, bulk velocity dispersion dominates the effective sound speed, while at large radii the thermal component dominates. Note that these are mass-weighted averages so the hot, low-density interarm medium does not contribute significantly to these profiles.

the two in-plane components of the velocity field account for exactly twice as much kinetic energy as the one out-of-plane component. In our model galaxies, the we find that this ratio is approximately 0.5 over the bulk of the disk, indicating that inplane motions contribute substantially more to the turbulent kinetic energy. This can also be seen in the thickness of our disks, where we typically find that the scale height is of order one or two cell spacings on the maximum refinement level. The extreme two-dimensional (2D) disks we form are likely an artifact of the lack of feedback in our simulations.

Finally, we show in the bottom panel of Figure 9 how the effective sound speed we measure in the inner regions of the gaseous disk is primarily due to bulk turbulent motions. The small sound speed we measure is typical of the dense gas in our simulation, which cools to temperatures of $\sim 200-300 \mathrm{~K}$ due to metal line cooling. The large velocity dispersions we measure indicates that the dense gas participates in large-scale turbulent motions.

\section{DISCUSSION AND CONCLUSIONS}

\subsection{Turbulence in a Gravity-dominated ISM}

Our simulations show, in agreement with previous numerical studies by Agertz et al. $(2009,2015)$ and Bournaud et al. (2010), that gravitational instability is capable of driving turbulence and stabilizing a disk galaxy at $Q \gtrsim 1$ even in the 
absence of any additional energy input from star formation. This state is characterized by a velocity dispersion that decreases somewhat with radius from $20 \mathrm{~km} \mathrm{~s}^{-1}$ near galactic centers to $10 \mathrm{~km} \mathrm{~s}^{-1}$ at large radii, in good agreement with observed gas velocity dispersions in nearby disk galaxies (Tamburro et al. 2009; Ianjamasimanana et al. 2015). Indeed, the only possibly significant disagreement between the kinematic behavior found in our simulations and that observed in nature is that our galaxies' velocity dispersions are anisotropic by a factor of $\sim 2$, leading to a scale height that is too small. Nonetheless, our results suggest that, even if stellar feedback is required to explain the vertical velocity distribution of the ISM, it need not be the dominant energy source for all turbulent motions. Gravity alone can generate the required inplane motion.

It is interesting to consider our simulations in light of the arguments commonly made that star formation feedback is the dominant driver of turbulence in galaxies. The primary observational argument for feedback driving turbulence is that there is a correlation between star formation rate and velocity dispersion, both within galaxies (Tamburro et al. 2009) and from galaxy to galaxy (Green et al. 2010). However, our simulations would also display precisely such a correlation: the regions of our galaxy that have the highest velocity dispersion are also the most strongly star-forming, and our models with the highest gas fractions show both the highest star formation rates and the highest velocity dispersions. The point is not that feedback cannot drive turbulence, simply that a correlation between star formation and turbulence does not necessarily imply a causal relationship between the two. A correlation of this sort can be produced even when there is no feedback.

The fact that galaxies can maintain $Q \gtrsim 1$ and continuously drive strong turbulence without star formation feedback calls into question analytic models in which galaxies' star formation rates are set by the need to maintain $Q=1$ (e.g., Thompson et al. 2005; Faucher-Giguère et al. 2013). We find that galaxies can maintain $Q \gtrsim 1$ regardless of the level of star formation feedback. Feedback is still needed to produce star formation rates in agreement with observation and drive out-of-plane motions, but the condition that determines the level of feedback and star formation appears to be completely decoupled from the need to maintain $Q \gtrsim 1$.

\subsection{Fueling Star Formation, From $z \sim 2$ to Today}

The primary result of our simulations is that gravitational instability-driven turbulence is capable of inducing significant bulk mass flows in galaxies, leading to a migration of mass inward from the passive outer regions of disks toward their actively star-forming centers. The inward mass transport rates we measure in our simulated galaxies are comparable to the star formation rates of typical $L_{*}$ galaxies. While there has been a great deal of work done on such inward migration in the context of the observed giant clumps in $z \sim 2$ galaxies (e.g., Genzel et al. 2008; Bournaud \& Elmegreen 2009; Cresci et al. 2009; Dekel et al. 2009b), our simulations show that a completely analogous phenomenon can operate for Milky Way-like galaxies at $z=0$.

An important implication of galaxies' ability to transport gas inward at rates comparable to their star formation rates is that their available fuel for star formation is their full gas reservoir, not simply the material in the actively star-forming inner disk. This is significant because, while gas depletion times are much less than the Hubble time only considering inner disk gas, the same is not true for many galaxies at $z=0$ if we consider the full gas reservoir. For a volume-limited sample of local starforming galaxies with stellar masses in the range $\log \left(M_{*} / M_{\odot}\right)=10-11.5$, Saintonge et al. (2011) find typical $\mathrm{H}$ I depletion times of $\sim 3 \mathrm{Gyr}$, with no strong dependence on stellar mass, and $\mathrm{H}_{2}$ depletion times that range from $\sim 0.5-3$ Gyr from the lowest to the highest stellar masses in the sample. If galaxies have access to their full extended $\mathrm{H}$ I reservoirs to fuel star formation, then their total depletion times are simply the sum of these, implying that galaxies with stellar masses above $\sim 10^{11} M_{\odot}$ have depletion times of $\sim 6 \mathrm{Gyr}$. This is still less than the Hubble time, but not by much. Galaxies in this mass range could receive no new gas supply after $z \sim 1$ and still fuel all their present star formation, particularly once the contribution from stellar recycling is included. Additionally, although galaxies with masses in the range $\log \left(M_{*} / M_{\odot}\right)=10-11$ have shorter molecular gas depletion times, the atomic gas depletion times are also $\sim 3$ Gyr. If these galaxies are also able to transport atomic gas from their outskirts into the active star-forming centers, they could supply all of their star formation without accreting any gas at all since $z \sim 0.5$.

We are therefore forced to conclude that strong equilibrium between gas inflow, star formation, and outflows may, by $z=0$, exist only for dwarf galaxies with $M_{*} \lesssim 10^{9.5} M_{\odot}$, since dwarfs are able to eject mass out of their star forming regions via high mass-loading factor winds. This means the mass range $M_{*} \sim 10^{9.5}-10^{10.5} M_{\odot}$, which corresponds to where galaxies are most efficient at converting baryons to stars (Behroozi et al. 2013), may not necessarily be in a state of nearinstantaneous equilibrium between gas accretion, star formation, and outflows. Galaxies with masses above $M_{*} \sim 10^{9.5} M_{\odot}$ may be far from equilibrium, contradicting one of the central assumptions of "bathtub" models of galaxy formation (e.g., Bouché et al. 2010; Lilly et al. 2013; Forbes et al. 2014b; Mitra et al. 2015).

This work utilized the Hyades supercomputer at the University of California Santa Cruz, which is supported by the National Science Foundation through award AST-1229745, and the Pleiades supercomputer, which is supported by the NASA Advanced Supercomputing Division. The computations and analysis described in this paper rely heavily on open source software packages, including Enzo, Python, yt, IPython, NumPy, SciPy, matplotlib, Cython, hdf5, h5py, and numexpr. We thank the developer communities of these packages for their contributions. This work was supported by NSF graduate fellowships (N.J.G. and J.C.F.), by NSF grants AST-0955300, AST-1405962 (M.R.K., N.J.G., and J.C.F.), and ACI-1535651 (N.J.G.), by NASA TCAN grant NNX14AB52G (M.R.K., N.J.G., and J.C.F.), by Hubble Archival Research grant HST-AR-13909 (J.C.F. and M.R.K.), and by the Gordon and Betty Moore Foundation's Data-Driven Discovert Initiative through Grant GBMF4651 to Matthew Turk.

\section{APPENDIX A ANALYSIS}

Here we describe the analysis pipeline used to post-process our simulations. 


\section{A.1. Grid Slabs}

Rather than analyzing the AMR data structures directly, we instead perform the bulk of our analysis using data interpolated onto uniform resolution meshes with $20 \mathrm{pc}$ cell spacings encompassing the galactic disk out to a radius of $20 \mathrm{kpc}$ and to a height of $1.25 \mathrm{kpc}$ above and below the disk. This choice significantly eases the implementation of the various analysis tasks we want to perform on the data without introducing a significant amount of error. Since the disk is very thin, a vertical extent of only $1.25 \mathrm{kpc}$ comfortably encloses the gas and stellar disk at all times.

To interpolate the gas data onto a uniform resolution grid, we make use of the smoothed covering grid object in yt. This operation uses cascading trilinear interpolation to represent an AMR data set at a uniform resolution. The fields representing gas density, the velocity vector components, and the gas thermal energy were extracted from the raw simulation outputs. Since the gravitational potential is not normally written to disk, we made use of the - $g$ command-line option of the Enzo code to solve the Poisson equation using the gravity in Enzo during post-processing.

So that we can apply the same analysis tasks we use for gas fields defined on Enzo's AMR mesh to $N$-body star particles in our data sets, we opt to analyze stellar fields by depositing the star particle data onto the uniform resolution grids slabs. In all cases, we use cloud-in-cell interpolation onto grids with the same shape and resolution as those used for the gas data. Our choice of uniform resolution slabs leads to issues with data sparseness in the outskirts of our simulated galaxies but is a good match for the density of particle data within a radius of $10 \mathrm{kpc}$.

\section{A.2. Rotation Curve and Epicyclic Frequency}

Because our simulations use a live stellar and dark matter halo, the rotation curve of our galaxy is not fixed, and must instead be computed self-consistently from the simulation outputs. Following Binney \& Tremaine (2008) and Shu (1992), we note that for an axisymmetric system with gravitational potential field $\Phi(R, z)$, we can define the circular frequency

$$
\Omega^{2}(R)=\frac{1}{R}\left(\frac{\partial \Phi}{\partial R}\right)_{(R, 0)},
$$

and epicyclic frequency

$$
\kappa^{2}(R)=\frac{2 \Omega}{R}\left(2 R \Omega+R^{2} \frac{d \Omega}{d R}\right) .
$$

This implicitly assumes the disk is thin so we can infer the circular velocity curve by only considering the gravitational potential in the midplane.

To measure the rotation frequency, we extract the gravitational potential in a slice at the midplane of the galaxy, and evaluate the partial derivative of the potential with respect to $x$ and $y$ coordinates using a centered finite difference of the gravitational potential. We then form a 2D array of the gradient with respect to the cylindrical $R$ coordinate out of the images of the $x$ and $y$ gradients. This results in a local estimate of the rotation frequency based on the local radial gradient in the gravitational potential. To average over local departures from axissymmetry, we create our final estimate of the rotation frequency by fitting a spline interpolator to a binned version of our local estimate of the circular frequency as a function of radius. This produces a binned $20 \mathrm{pc}$ resolution estimate of the rotation frequency as a function only of cylindrical radius. Finally, we calculate the circular velocity via

$$
v_{c}=R \Omega \text {. }
$$

\section{A.3. Surface Density}

Several different quantities we are concerned with are defined in terms of the projection of our simulation data. For a 3D density field $\rho(x, y, z)$ we can define the surface density,

$$
\Sigma(x, y)=\int_{-\infty}^{\infty} \rho d z .
$$

Here $\rho$ represents the mass density of gas or stars, which we denote as $\rho_{\text {gas }}$ and $\rho_{*}$ below. Both quantities are defined on the uniform resolution grid slabs discussed in Appendix A.1.

Discretizing $\rho$ into a uniform resolution 3D array, which we denote as $\rho_{i j k}$, the continuous definition of the surface density reduces to

$$
\Sigma_{i j}=\sum_{k=0}^{N_{z}} \rho_{i j k} \Delta z,
$$

where $\Delta z$ is the cell spacing the vertical direction. Since our grid slabs only include data within $1.25 \mathrm{kpc}$ of the disk midplane, we are implicitly assuming that gas well off the midplane does not contribute significantly to the surface density.

We introduce the notation, $\langle q\rangle_{z}$, to represent the discrete mass-weighted projection operator. The result is a uniform resolution $2 \mathrm{D}$ array, where the $i, j$ resolution element can be found by computing,

$$
\langle q\rangle_{z, i j}=\frac{1}{\sum_{i j}} \sum_{k=0}^{N_{z}} \rho_{i j k} q_{i j k} \Delta z .
$$

This notation allows us to write several of the definitions below in a compact form.

\section{A.4. Velocity Dispersion}

Since turbulent motions are a significant component of the energy budget in the ISM, we would like to directly measure the turbulent kinetic energy in our simulations. Since our simulations are a discretized representation of a continuous underlying system, we can only estimate the velocity dispersion by comparing velocity values in cells contained within a moving window several cells across. So that variations in the rotation curve over the extent of the window do not tend to inflate the measured velocity dispersions, we define the velocity of streaming motions

$$
\delta \boldsymbol{v}=\boldsymbol{v}-v_{c}(R) \hat{\phi} .
$$

To simplify notation in our definition of the velocity dispersion, we define the discrete operator

$$
C_{i j k}(x)=\sum_{k^{\prime}=k-2 j^{\prime}=j-2 i^{\prime}=i-2}^{k+2} \sum_{i^{\prime} j^{\prime} k^{\prime}}^{j+2},
$$

where $x$ is a field defined on the uniform resolution mesh. This is equivalent to a discrete convolution of $x$ using a cubical tophat kernel function. The top-hat kernel includes 5 cells, so the spatial scale of the convolution is $100 \mathrm{pc}$. Using this notation, 
our estimate of the velocity dispersion due to motions in the $x$ coordinate, $\delta v_{x}$, can be written

$$
\sigma_{x, i j k}=\frac{\sqrt{C(\rho) C\left(\rho \delta v_{x}^{2}\right)-C\left(\rho \delta v_{x}\right)^{2}}}{C(\rho)},
$$

where $\delta v_{x}$ is the $x$ component of the streaming velocity vector $\delta \mathbf{v}$. The expressions for the $y$ and $z$ velocity dispersions are analogous. We write the variance in terms of the convolution operator since this form lends itself to faster one-pass parallel reduction. This result can be derived from the definition of the weighted variance for a sampled quantity with measurements $x_{i}$ and weights $w_{i}$, and weighted mean $\bar{x}_{w}$, $\sigma^{2}=\sum_{i=1}^{N} w_{i}\left(x_{i}-\bar{x}_{w}\right)^{2} / \sum_{i=1}^{N} w_{i}$ by expanding the squared term and substituting for the definition of the weighted mean.

We use the same definition for stellar velocity dispersions, but rather than calculating the dispersion on a cell-by-cell basis, we instead iterate over particles, performing a running dispersion calculation by depositing particle densities and velocities into accumulating arrays. This uses more memory, but is substantially faster for the particle counts in our simulations. Like the gas velocity dispersions, the stellar velocity dispersion is computed on a spatial scale of $100 \mathrm{pc}$.

Finally, we calculate the projected velocity dispersions by performing a weighted projection of the turbulent kinetic energy density:

$$
\sigma_{v}=\sqrt{\left\langle\sigma_{x}^{2}+\sigma_{y}^{2}+\sigma_{z}^{2}\right\rangle_{z}} .
$$

In addition, to estimate the relative contribution of in-plane and out-of-plane turbulent motions, we separately define the inplane velocity dispersion

$$
\sigma_{v, d}=\sqrt{\left\langle\sigma_{x}^{2}+\sigma_{y}^{2}\right\rangle_{z}},
$$

and the out-of-plane velocity dispersion

$$
\sigma_{v, z}=\sqrt{\left\langle\sigma_{z}^{2}\right\rangle_{z}} \text {. }
$$

\section{A.5. Effective Sound Speed}

We can define an effective sound speed that takes into account both gas pressure and turbulent pressure,

$$
c_{\text {eff }}=\sqrt{\sigma_{v}^{2}+c_{s}^{2}}
$$

For collisionless fluids, $c_{\text {eff }}=\sigma_{v}$, but for the gaseous component we must calculate the sound speed. In practice, we do this in terms of the weighted projection of the thermal energy density $e$,

$$
c_{s}=\sqrt{\gamma(\gamma-1)\langle e\rangle_{z}}
$$

where $\gamma=5 / 3$ is the adiabatic index.

\section{A.6. Toomre Q}

Using the derived data we introduced above, we can compute the Toomre $Q$ parameter for both the gas,

$$
Q_{\text {gas }}=\frac{c_{\text {eff }} \kappa}{\pi G \Sigma_{\text {gas }}}
$$

and stars,

$$
Q_{*}=\frac{\sigma_{v, *} \kappa}{3.36 G \Sigma_{*}} .
$$

We also attempt to estimate the degree of gravitational instability due to both the gas and stars. We make use of the formula derived by Romeo \& Wiegert (2011) that takes into account the separate contribution of the gas and stars as well as their finite thickness. In this formalism,

$$
\frac{1}{Q_{\mathrm{total}}}=\left\{\begin{array}{ll}
\frac{W}{T_{*} Q_{*}}+\frac{1}{T_{\mathrm{gas}} Q_{\mathrm{gas}}}: T_{*} Q_{*} \geqslant T_{\mathrm{gas}} Q_{\mathrm{gas}} \\
\frac{1}{T_{*} Q_{*}}+\frac{W}{T_{\mathrm{gas}} Q_{\mathrm{gas}}}: T_{*} Q_{*}<T_{\mathrm{gas}} Q_{\mathrm{gas}}
\end{array},\right.
$$

where $T=0.8+0.7 \sigma_{z} / \sigma_{d}$, and $W=2 \sigma_{*} \sigma_{\text {gas }} /\left(\sigma_{*}^{2}+\sigma_{\text {gas }}^{2}\right)$.

\section{A.7. Scale Height}

For a fluid with local mass density $\rho$, we define the scale height $h$ such that

$$
\int_{0}^{h} \rho d z=\left(1-e^{-1}\right) \int_{0}^{\infty} \rho d z .
$$

Here the $z$ coordinate is perpendicular to the disk and centered on the disk midplane. Since the fluid distribution is not necessarily symmetric in $z$, the $z$ coordinate of the midplane is not constant, and the scale height above and below the midplane might not be equal. In practice, we take the true scale height $h$ to be the arithmetic mean of the scale heights measured above and below the midplane.

We generate maps of of the scale height by individually processing $z$-aligned pencil stacks of cells. We calculate a running sum of the gas mass along each pencil stack and note the $z$ locations where the surface density equals one of $(2 e)^{-1} \Sigma$, $\Sigma / 2$, and $\left(1.0-(2 e)^{-1}\right) \Sigma$. We use linear interpolation in $z$ to estimate the intra-cell locations where the running sum exceeds each critical value. Finally, we calculate a single scale height estimate by averaging the "top" and "bottom" estimates.

\section{A.8. Radial Mass Flux}

For a cylindrical test volume $V$ of radius $R$, the flux of mass across the surface of the cylinder is

$$
\dot{M}=R \int_{0}^{2 \pi} \int_{-\infty}^{\infty} \rho v_{r} d z d \theta .
$$

In practice, we calculate this quantity using a discrete approximation based on our interpolated data defined on uniform resolution grid slabs. Here we can take advantage of the cylindrical symmetry of the problem to substantially reduce the computational cost of this calculation. If we consider a single $x-y$ slice through our grid slab, it is a straightforward geometric problem to find the set of cells in this slice that the cylindrical test volume intersects.

If we define a one-dimensional index $l \in[0, N]$ where $N$ is the total number of cells in the $x-y$ plane that intersect with the circular test region, we can approximate

$$
\dot{M} \approx \sum_{k=0}^{k_{\max }} \sum_{l=0}^{N} \rho_{l k} v_{r, l k} \Delta \theta_{l} \Delta z .
$$


Here $l$ maps to a set of unique coordinates in the 2D slice of the $3 \mathrm{D}$ grid slab and $k$ indexes along the $z$ direction in the grid slab. One can think of $l$ as a map to a single pair of $i, j$ indices for the $x$ and $y$ rows in our grid slice. The problem of calculating the radial mass flux reduces to calculating the angles subtended by each cell $\Delta \theta_{l}$. Since we ignore gas well off the midplane, this implicitly assumes that the mass transport at large heights above and below the midplane is small.

Doing this at many different radii allows up to map how the radial mass flux varies with galactocentric radius. In practice, we choose a set of 1000 nested test cylinders, binned evenly in radius with a spacing of $40 \mathrm{pc}$.

\section{A.9. Time Averaging}

We are interested in the time-average behavior of azimuthally averaged quantities, both to compare to 1D models of galaxy formation and also to capture the large-scale behavior of our simulations over long periods of time. As we show in the main text, the internal structure of our simulated galaxies is initially dominated by transient disturbances. After several galactic rotation periods, the disks settle down and reach a quasi-equilibrium state in which the global structure is more or less static. Since the galactic rotation period is an increasing function of radius, the places where we expect the disk to be settled corresponds to a wedge-shaped region of radius-time phase space.

For any azimuthally averaged quantity $x(r, t)$, we define its time average, a function of radius only, as

$$
\langle x(r, t)\rangle_{t}=\frac{1}{t_{\max }-\left(t_{0}+a r\right)} \int_{t_{0}+a r}^{t_{\max }} x(r, t) d t,
$$

where $t_{\max }$ is the simulation time at the end of the simulation (600 Myr for all three simulations presented here) and $a$ and $t_{0}$ are constants that define the shape of the "averaging wedge" as denoted by the blue lines in Figure 6 . In practice we determine these constants "by eye" based on inspection of the simulation results.

\section{REFERENCES}

Agertz, O., Lake, G., Teyssier, R., et al. 2009, MNRAS, 392, 294 Agertz, O., Romeo, A. B., \& Grisdale, K. 2015, MNRAS, 449, 2156 Balser, D. S., Rood, R. T., Bania, T. M., \& Anderson, L. D. 2011, ApJ, 738, 27 Behrendt, M., Burkert, A., \& Schartmann, M. 2015, MNRAS, 448, 1007 Behroozi, P. S., Wechsler, R. H., \& Conroy, C. 2013, ApJL, 762, L31

Bertin, G., \& Lodato, G. 1999, A\&A, 350, 694

Bertin, G., \& Romeo, A. B. 1988, A\&A, 195, 105

Bigiel, F., \& Blitz, L. 2012, ApJ, 756, 183

Bigiel, F., Leroy, A., Walter, F., et al. 2008, AJ, 136, 2846

Binney, J., \& Tremaine, S. 2008, Galactic Dynamics (2nd ed.; Princeton, NJ: Princeton Univ. Press)

Bouché, N., Dekel, A., Genzel, R., et al. 2010, ApJ, 718, 1001

Bournaud, F., \& Elmegreen, B. G. 2009, ApJL, 694, L158

Bournaud, F., Elmegreen, B. G., Teyssier, R., Block, D. L., \& Puerari, I. 2010, MNRAS, 409, 1088

Bresolin, F., Kennicutt, R. C., \& Ryan-Weber, E. 2012, ApJ, 750, 122

Bresolin, F., Ryan-Weber, E., Kennicutt, R. C., \& Goddard, Q. 2009, ApJ, 695,580

Bryan, G. L., Norman, M. L., Stone, J. M., Cen, R., \& Ostriker, J. P. 1995, $\mathrm{CoPhC}, 89,149$

Cen, R., \& Ostriker, J. P. 1992, ApJL, 399, L113

Chomiuk, L., \& Povich, M. S. 2011, AJ, 142, 197

Colella, P., \& Woodward, P. R. 1984, JCoPh, 54, 174

Cresci, G., Hicks, E. K. S., Genzel, R., et al. 2009, ApJ, 697, 115

Croxall, K. V., van Zee, L., Lee, H., et al. 2009, ApJ, 705, 723
Daddi, E., Dickinson, M., Morrison, G., et al. 2007, ApJ, 670, 156

Dekel, A., Birnboim, Y., Engel, G., et al. 2009a, Natur, 457, 451

Dekel, A., Sari, R., \& Ceverino, D. 2009b, ApJ, 703, 785

Dobbs, C. L., Bonnell, I. A., \& Pringle, J. E. 2006, MNRAS, 371, 1663

Dobbs, C. L., Pringle, J. E., \& Duarte-Cabral, A. 2015, MNRAS, 446, 3608

Draine, B. T. 2011, Physics of the Interstellar and Intergalactic Medium (Princeton, NJ: Princeton Univ. Press)

Dutton, A. A. 2012, MNRAS, 424, 3123

Elbaz, D., Daddi, E., Le Borgne, D., et al. 2007, A\&A, 468, 33

Elmegreen, B. G., \& Hunter, D. A. 2015, ApJ, 805, 145

Faucher-Giguère, C.-A., Quataert, E., \& Hopkins, P. F. 2013, MNRAS, 433, 1970

Ferguson, A. M. N., \& Clarke, C. J. 2001, MNRAS, 325, 781

Forbes, J., Krumholz, M., \& Burkert, A. 2012, ApJ, 754, 48

Forbes, J. C., Krumholz, M. R., Burkert, A., \& Dekel, A. 2014a, MNRAS, 438, 1552

Forbes, J. C., Krumholz, M. R., Burkert, A., \& Dekel, A. 2014b, MNRAS, 443, 168

Fraternali, F., Marasco, A., Marinacci, F., \& Binney, J. 2013, ApJL, 764, L21

Fujimoto, Y., Tasker, E. J., \& Habe, A. 2014a, MNRAS, 445, L65

Fujimoto, Y., Tasker, E. J., Wakayama, M., \& Habe, A. 2014b, MNRAS, 439, 936

Genzel, R., Burkert, A., Bouché, N., et al. 2008, ApJ, 687, 59

Green, A. W., Glazebrook, K., McGregor, P. J., et al. 2010, Natur, 467, 684

Guedes, J., Callegari, S., Madau, P., \& Mayer, L. 2011, ApJ, 742, 76

Henry, R. B. C., Kwitter, K. B., Jaskot, A. E., et al. 2010, ApJ, 724, 748

Hernquist, L. 1990, ApJ, 356, 359

Ho, I.-T., Kudritzki, R.-P., Kewley, L. J., et al. 2015, MNRAS, 448, 2030

Hobbs, A., Read, J., Power, C., \& Cole, D. 2013, MNRAS, 434, 1849

Hockney, R. W., \& Eastwood, J. W. 1988, Computer Simulation Using Particles (Boca Raton, FL: CRC Press)

Hopkins, P. F., Quataert, E., \& Murray, N. 2012, MNRAS, 421, 3488

Ianjamasimanana, R., de Blok, W. J. G., Walter, F., \& Heald, G. H. 2012, AJ, 144, 96

Ianjamasimanana, R., de Blok, W. J. G., Walter, F., et al. 2015, AJ, 150, 47

Jog, C. J., \& Solomon, P. M. 1984a, ApJ, 276, 127

Jog, C. J., \& Solomon, P. M. 1984b, ApJ, 276, 114

Kennicutt, R. C., \& Evans, N. J. 2012, ARA\&A, 50, 531

Kereš, D., Katz, N., Weinberg, D. H., \& Davé, R. 2005, MNRAS, 363, 2

Kim, J.-h., Abel, T., Agertz, O., et al. 2014, ApJS, 210, 14

Kim, W., Ostriker, E. C., \& Stone, J. M. 2003, ApJ, 599, 1157

Krumholz, M., \& Burkert, A. 2010, ApJ, 724, 895

Krumholz, M. R., Dekel, A., \& McKee, C. F. 2012, ApJ, 745, 69

Krumholz, M. R., \& Tan, J. C. 2007, ApJ, 654, 304

Leitner, S. N., \& Kravtsov, A. V. 2011, ApJ, 734, 48

Leroy, A. K., Walter, F., Brinks, E., et al. 2008, AJ, 136, 2782

Leroy, A. K., Walter, F., Sandstrom, K., et al. 2013, AJ, 146, 19

Lilly, S. J., Carollo, C. M., Pipino, A., Renzini, A., \& Peng, Y. 2013, ApJ, 772,119

Lin, C. C., \& Shu, F. H. 1966, PNAS, 55, 229

Lin, D. N. C., \& Pringle, J. E. 1987a, MNRAS, 225, 607

Lin, D. N. C., \& Pringle, J. E. 1987b, ApJL, 320, L87

Marinacci, F., Binney, J., Fraternali, F., et al. 2010, MNRAS, 404, 1464

Mitra, S., Davé, R., \& Finlator, K. 2015, MNRAS, 452, 1184

Mo, H. J., Mao, S., \& White, S. D. M. 1998, MNRAS, 295, 319

Moster, B. P., Naab, T., \& White, S. D. M. 2013, MNRAS, 428, 3121

Navarro, J. F., Frenk, C. S., \& White, S. D. M. 1996, ApJ, 462, 563

Noeske, K. G., Weiner, B. J., Faber, S. M., et al. 2007, ApJL, 660, L43

Olivier, S. S., Primack, J. R., \& Blumenthal, G. R. 1991, MNRAS, 252, 102

Petit, A. C., Krumholz, M. R., Goldbaum, N. J., \& Forbes, J. C. 2015, MNRAS, 449, 2588

Petric, A. O., \& Rupen, M. P. 2007, AJ, 134, 1952

Pilyugin, L. S., Vílchez, J. M., \& Contini, T. 2004, A\&A, 425, 849

Piontek, R. A., \& Ostriker, E. C. 2004, ApJ, 601, 905

Piontek, R. A., \& Ostriker, E. C. 2005, ApJ, 629, 849

Piontek, R. A., \& Ostriker, E. C. 2007, ApJ, 663, 183

Portinari, L., \& Chiosi, C. 2000, A\&A, 355, 929

Regan, M. W., Thornley, M. D., Helfer, T. T., et al. 2001, ApJ, 561, 218

Renaud, F., Bournaud, F., Emsellem, E., et al. 2013, MNRAS, 436, 1836

Romeo, A. B., \& Wiegert, J. 2011, MNRAS, 416, 1191

Saintonge, A., Kauffmann, G., Wang, J., et al. 2011, MNRAS, 415, 61

Schruba, A., Leroy, A. K., Walter, F., et al. 2011, AJ, 142, 37

Shu, F. H. 1992, Physics of Astrophysics, Vol. II (Mill Valley, CA: Univ. Science Books)

Smith, R. J., Glover, S. C. O., Clark, P. C., Klessen, R. S., \& Springel, V. 2014 MNRAS, 441, 1628 
Spitoni, E., \& Matteucci, F. 2011, A\&A, 531, A72

Springel, V., Di Matteo, T., \& Hernquist, L. 2005, MNRAS, 361, 776

Stilp, A. M., Dalcanton, J. J., Skillman, E., et al. 2013, ApJ, 773, 88

Tamburro, D., Rix, H.-W., Leroy, A. K., et al. 2009, AJ, 137, 4424

Tasker, E. J. 2011, ApJ, 730, 11

Tasker, E. J., \& Tan, J. C. 2009, ApJ, 700, 358

Tasker, E. J., Wadsley, J., \& Pudritz, R. 2015, ApJ, 801, 33

The Enzo Collaboration Bryan, G. L., Norman, M. L., O’Shea, B. W., et al. 2014, ApJS, 211, 19

Thompson, T. A., Quataert, E., \& Murray, N. 2005, ApJ, 630, 167

Toomre, A. 1964, ApJ, 139, 1217
Tremonti, C. A., Heckman, T. M., Kauffmann, G., et al. 2004, ApJ, 613,898

Truelove, J. K., Klein, R. I., McKee, C. F., et al. 1998, ApJ, 495, 821

Turk, M. J., Smith, B. D., Oishi, J. S., et al. 2011, ApJS, 192, 9

van Zee, L., \& Bryant, J. 1999, AJ, 118, 2172

Vila-Costas, M. B., \& Edmunds, M. G. 1992, MNRAS, 259, 121

Wang, B., \& Silk, J. 1994, ApJ, 427, 759

Werk, J. K., Putman, M. E., Meurer, G. R., \& Santiago-Figueroa, N. 2011 , ApJ, 735, 71

Yang, C.-C., Gruendl, R. A., Chu, Y.-H., Mac Low, M.-M., \& Fukui, Y. 2007, ApJ, 671, 374 